\title{
Gyration-radius dynamics in structural transitions of atomic clusters
}

\author{
Tomohiro Yanao, ${ }^{\text {a) }}$ Wang S. Koon, and Jerrold E. Marsden \\ Control and Dynamical Systems, MC 107-81, California Institute of Technology, Pasadena, \\ California 91125 \\ loannis G. Kevrekidis \\ Department of Chemical Engineering, Princeton University, Princeton, New Jersey 08544 \\ and PACM, Princeton University, Princeton, New Jersey 08544
}

(Received 23 June 2006; accepted 25 January 2007; published online 22 March 2007)

\begin{abstract}
This paper is concerned with the structural transition dynamics of the six-atom Morse cluster with zero total angular momentum, which serves as an illustrative example of the general reaction dynamics of isolated polyatomic molecules. It develops a methodology that highlights the interplay between the effects of the potential energy topography and those of the intrinsic geometry of the molecular internal space. The method focuses on the dynamics of three coarse variables, the molecular gyration radii. By using the framework of geometric mechanics and hyperspherical coordinates, the internal motions of a molecule are described in terms of these three gyration radii and hyperangular modes. The gyration radii serve as slow collective variables, while the remaining hyperangular modes serve as rapidly oscillating "bath" modes. Internal equations of motion reveal that the gyration radii are subject to two different kinds of forces: One is the ordinary force that originates from the potential energy function of the system, while the other is an internal centrifugal force. The latter originates from the dynamical coupling of the gyration radii with the hyperangular modes. The effects of these two forces often counteract each other: The potential force generally works to keep the internal mass distribution of the system compact and symmetric, while the internal centrifugal force works to inflate and elongate it. Averaged fields of these two forces are calculated numerically along a reaction path for the structural transition of the molecule in the three-dimensional space of gyration radii. By integrating the sum of these two force fields along the reaction path, an effective energy curve is deduced, which quantifies the gross work necessary for the system to change its mass distribution along the reaction path. This effective energy curve elucidates the energy-dependent switching of the structural preference between symmetric and asymmetric conformations. The present methodology should be of wide use for the systematic reduction of dimensionality as well as for the identification of kinematic barriers associated with the rearrangement of mass distribution in a variety of molecular reaction dynamics in vacuum. (C) 2007 American Institute of Physics. [DOI: 10.1063/1.2710272]
\end{abstract}

\section{INTRODUCTION}

Structural transition dynamics of molecules, clusters, and biopolymers are of fundamental interest in current chemistry and biophysics research. The high-dimensional nature of the dynamics is often the main obstacle to clarifying the exact mechanisms of such intriguing collective motions. Reducing the dimensionality is therefore crucial and has been a longstanding issue in nonequilibrium statistical mechanics, ${ }^{1-3}$ molecular dynamics, ${ }^{4-6}$ Monte Carlo simulations, ${ }^{7}$ and in reaction path theories. ${ }^{8-10}$ In order to carry out the dimensionality reduction effectively, it is crucial to identify a small number of collective (or coarse) variables that play a predominant role in the structural dynamics of molecules. The low-dimensional dynamics of such collective variables should pave the way to the true understanding of large-amplitude structural transitions.

As collective variables, radial variables of molecules such as the radii of gyration and the principal moments of

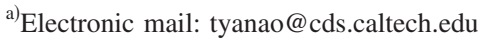

inertia are widely used. For example, the one-dimensional averaged radius of gyration has long been used as an important order parameter to quantify size changes of polymers ${ }^{11-14}$ and protein folding. ${ }^{15,16}$ The so-called asphericity parameter, which is defined in terms of the three principal moments of inertia, has also been employed to characterize morphological changes of DNA-like polymers. ${ }^{17}$ Rotational constants are also used to characterize the deformation of rotating clusters. ${ }^{18,19}$ These examples clearly demonstrate the usefulness of radial variables as good collective variables. Although many past studies utilize such radial variables in statistical or phenomenological arguments, dynamical study of these variables at the level of their intrinsic equations of motion provides a complementary basis for the elucidation of the mechanism of large-amplitude motions of complex molecules as well as for their dimensionality reduction.

This paper focuses on the dynamical principles and associated equations of motion of the molecular gyration radii that are inherent in the dynamics of isolated polyatomic molecules. In contrast to the conventional statistical approaches 
using a single (averaged) radius of gyration mentioned above, we treat here the three gyration radii of the system on an equal footing as the important collective variables. By using the three gyration radii, the structural transitions of molecules are viewed as rearrangements of the mass distribution. Whenever the system changes its mass distribution significantly in large-amplitude motions, the three gyration radii can potentially serve as collective variables. We then address a basic question of what is the necessary work or energy for the system to change its mass distribution against the forces acting on the gyration radii. This work can in turn elucidate the effective barrier structure in the structural transitions of the system. Here, the dynamics of the three gyration radii is regarded as the most essential slow part of the full dynamics of the polyatomic molecule.

For an isolated $n$-atom molecule, we begin with the $3 n$ total degree of freedom configuration space and by systematically reducing the dimensionality, we arrive at a reduced system in terms of three gyration radii. Using standard reduction theory for symmetric systems following Refs. 20 and 21 and the associated gauge theory, ${ }^{22-27}$ the three rotational degrees of freedom are first separated out from the $(3 n-3)$ degrees of freedom after elimination of the three overall translational ones. This is a vital first step for the rigorous investigation of the internal (shape) dynamics of molecules. A remarkable consequence of these theories is that the $(3 n$ -6)-dimensional internal space is equipped with nonEuclidean properties which give rise to important massrelated kinematic effects. As will be shown, these kinematic effects can be compactly extracted in the reduced dynamics of gyration radii and they turn out to be comparable in importance with, or even overshadow, the effects of the potential energy barriers.

In order to carry on further reduction in dimensions, beyond symmetry reduction, we employ the principal-axis hyperspherical formalism, which was initiated in an early paper by Eckart $^{28}$ and reformulated in the hyperspherical context by Chapuisat et $a l .^{29,30}$ This coordinate system is also known as the row-orthonormal hyperspherical coordinates, developed by Kuppermann. ${ }^{31-33}$ The principal axes of the system are used as a body frame, which determines the orientation of the system at each instant. Then the $(3 n-6)$ internal degrees of freedom can be parametrized by the three gyration radii and the $(3 n-9)$ hyperangles. A dynamical change in the hyperangles is called a kinematic or democratic rotation ${ }^{34-37}$ which is essentially a continuous and cyclic shape change. The kinematic rotations are the "rotations" in the internal space, whose representative group is a subgroup of $\mathrm{SO}(n-1)$. They should be clearly distinguished from the (external) rotations $[\mathrm{SO}(3)]$ in physical space. Littlejohn et $a l{ }^{35-37}$ clarified the geometry of kinematic rotations in the few-body problem. Aquilanti et al. $^{38-40}$ investigated roles of the conjugate "angular momenta" of the kinematic rotations in the critical behavior of atomic clusters. As was shown in Refs. 28-30, the use of $(3 n-9)$ quasivelocities instead of the hyperangles simplifies the expression of kinetic energy remarkably. These quasivelocities are physically the "angular velocities" of the kinematic rotations. Thanks to the simplic- ity of the kinetic energy expression, the resulting classical equations of motion are also concise and physically appealing.

The equations of motion for the gyration radii shed light on the mechanism of competition between the potential force and an internal kinematic force in the structural dynamics of polyatomic molecules. The kinematic force has its origin in the dynamical coupling of gyration radii with the hyperangular modes (kinematic rotations) via the non-Euclidean metric of the internal space. This kinematic force has a quadratic dependence on the quasivelocity components of the hyperangular modes. Therefore it is essentially an internal centrifugal force arising from the internal motions themselves. Generally speaking, the potential force works to keep the internal mass distribution of a molecule symmetric and compact. On the other hand, the internal centrifugal force has the remarkable effect of elongating and inflating the internal mass distribution of the molecule. In other words, the molecular vibrations induce a spontaneous tendency of deformation. In this way, the internal centrifugal force can be the driving force for a molecule to move from one potential well to another.

Effects of the internal centrifugal force in three-atom and four-atom reactions have been studied in Refs. 41-43. Amongst other things, the present study generalizes these results to larger $n$-atom systems through systematic averaging methods. The three gyration radii are regarded as slow collective variables while the hyperangular modes are regarded as fast "bath" modes-this distinction is based on numerical observations of time scale separation. Based on this observation, the dependence of the equations of motion for the gyration radii on the hyperangular modes is numerically averaged. This procedure is analogous to the adiabatic approximation used in the stability arguments of few-body quantum systems ${ }^{44,45}$ as well as of Bose-Einstein condensates. ${ }^{46,47}$ As a result, the averaged force fields both for the potential force and the internal centrifugal force are quantified and depicted in the three-dimensional space of gyration radii. A one-dimensional reaction path is also deduced in this three-dimensional space by directly numerically averaging the real trajectories. The directionality of the averaged force fields with respect to the path indicates the preferred reaction direction. We then deduce an effective energy curve along the reaction path, which is a quantification of the "work" necessary for the system to change its mass distribution along the path. This energy curve, which can differ significantly from the potential energy curve along the path, characterizes the true barrier structure for the reaction.

The illustrative model system of this study is a six-atom Morse cluster, which represents an $\mathrm{Ar}_{6}$-like cluster, with constant energy and with zero total angular momentum. The potential energy surface of the $\mathrm{Ar}_{6}$-like cluster has been extensively studied by Berry and co-workers. ${ }^{48,49}$ Komatsuzaki and Berry ${ }^{50}$ scrutinized the regularity of the saddle crossing motions of the $\mathrm{Ar}_{6}$-like cluster. The cluster possesses two kinds of geometrically distinct isomers, one of which has a regular octahedron structure, which is highly symmetric, while the other has an elongated structure. The octahedron structure lies at the bottom of a much deeper potential well. 
Interestingly, this cluster shows a clear switching of structural preference depending on its total energy. In the low energy range (solidlike phase), the cluster spends more time in the potential well of the octahedron structure, while in the high energy range (liquidlike phase), the cluster spends more time in the competing potential well. This kind of two-state switching is interesting in view of two-state first-order phase transitions of clusters ${ }^{51-53}$ and of polymers. ${ }^{54,55}$

From the viewpoint of statistical reaction rate theory, the switching of structural preference can be explained in terms of the densities of states (phase space volumes) for respective isomers. ${ }^{56-58}$ However, this statistical approach does not explain the dynamical origin of the structural preference. In addition, an accurate computation of the density of states is often a hard task, especially for large systems. Our approach provides a different explanation for such switching in terms of the reduced dynamics of gyration radii: Since the internal centrifugal force originates from the internal kinetic energy, its elongation and inflation effects generally become more prominent in the high energy range. Therefore, the octahedron isomer, which has an isotropic mass distribution (like a spherical top), will not be preferred, especially in the high energy regime.

The paper is organized as follows. In Sec. II, the model system, the six-atom Morse cluster, is introduced. We then characterize the isomerization dynamics of the cluster using gyration radii as collective variables. In Sec. III, the principal-axis hyperspherical coordinates are introduced, and the intrinsic equations of motion for gyration radii of isolated $n$-atom systems are investigated in detail. The properties of the kinematic force in the reduced dynamics of gyration radii are highlighted. In Sec. IV, the competition between this kinematic force and the potential force is characterized by averaging these forces and by introducing an effective energy along a reaction path defined in the space of gyration radii. The structural preference of the cluster is finally accounted for in terms of this effective energy. The paper concludes with some remarks and a discussion of future directions in Sec. V.

\section{CHARACTERIZATION OF ISOMERIZATION DYNAMICS OF A SIX-ATOM CLUSTER USING GYRATION RADII}

\section{A. Model system: $\mathrm{M}_{6}$ cluster}

In this paper, we study, as a prototypical example, the structural isomerization dynamics of a cluster composed of six identical atoms that mutually interact through a pairwise Morse potential; this is called an $\mathrm{M}_{6}$ cluster. The dimensionless Hamiltonian of the system is given by

$$
\frac{\mathcal{H}}{\varepsilon}=\frac{1}{2} \sum_{i=1}^{6}\left(\dot{\boldsymbol{r}}_{s i} \cdot \dot{\boldsymbol{r}}_{s i}\right)+\sum_{i<j}\left[e^{-2\left(d_{i j}-d_{0}\right)}-2 e^{-\left(d_{i j}-d_{0}\right)}\right]
$$

where $\boldsymbol{r}_{s i}(i=1, \ldots, 6)$ is the three-dimensional position vector of the atom $i$. The subscript $s$ represents the quantity with respect to the space-fixed frame. (This rule is also applied to other quantities.) The dot over $\boldsymbol{r}_{s i}$ represents the time derivative. All the masses of the atoms are set to unity, $m_{i}=1(i$ $=1, \ldots, 6)$. The parameter $\varepsilon$ represents the depth of the

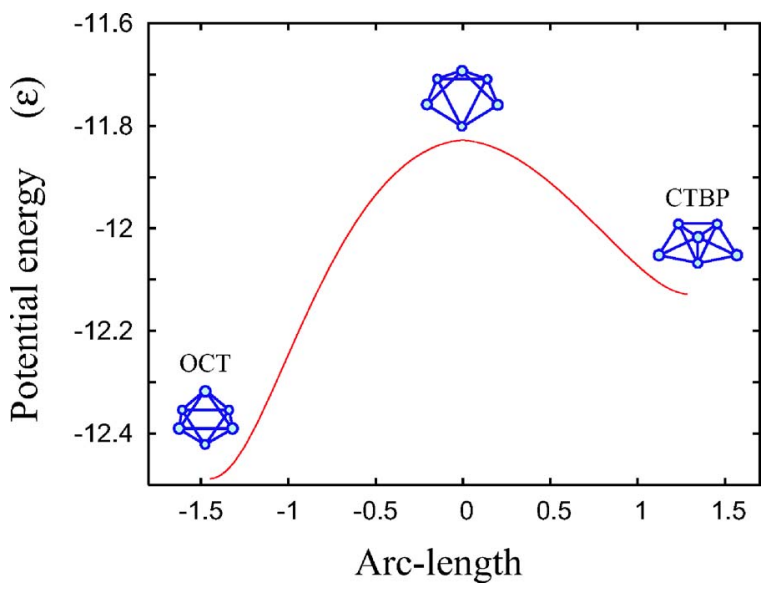

FIG. 1. (Color online) Isomerization scheme of the six-atom Morse cluster. The cluster has two geometrically distinct isomers, OCT and CTBP. The potential energy curve along the steepest descent path connecting the saddle point and the two potential minima is shown. The potential energy of the OCT isomer at the minimum point is $V=-12.49 \varepsilon$, while that of the CTBP isomer is $V=-12.13 \varepsilon$. These minima are connected through a saddle point whose potential energy is $V=-11.83 \varepsilon$.

Morse potential and $d_{i j}$ is the interparticle distance between atom $i$ and atom $j$. The parameter $d_{0}$, which corresponds to the equilibrium distance of the pairwise Morse potential, is set to $d_{0}=6.0$. This provides a potential topography similar to that of the Lennard-Jones potential. ${ }^{49}$ Therefore, this system can be regarded as a model of the $\mathrm{Ar}_{6}$ cluster. Since the main interest of this study is in the internal dynamics of polyatomic systems, the total angular momentum of the system is assumed to be zero throughout the paper. In what follows, our numerical results are presented in absolute units.

The isomerization scheme of the $\mathrm{M}_{6}$ cluster is shown in Fig. 1. This cluster has two geometrically distinct isomers: One is the regular octahedron (OCT) and the other is the capped trigonal bipyramid (CTBP). ${ }^{48-50}$ The potential energy minimum of the OCT isomer is $V=-12.49 \varepsilon$ and that of the CTBP isomer is $V=-12.13 \varepsilon$. These two isomers are connected through a saddle point whose potential energy is $V=$ $-11.83 \varepsilon$. In Fig. 1, the potential energy topography along the steepest descent path is shown. The horizontal axis is the arc length of the path in the space of gyration radii, which will be explained later. Note that the OCT isomer has a highly symmetric structure (that of a spherically symmetric rigid body) with a deep potential well, while the CTBP isomer has an elongated (collapsed) structure with a shallow potential well.

A microcanonical, constant energy simulation is employed. If the total energy of the cluster is higher than that of the saddle point, the isomerization reaction between the two isomers is energetically possible. Occurrence of the structural isomerization reaction is detected by the quenching method $^{48}$ (that is, a descent method), which solves the first order equations,

$$
\frac{d \boldsymbol{r}_{s i}}{d \tau}=-\frac{\partial V}{\partial \boldsymbol{r}_{s i}},
$$

at each instant until the system arrives at one of the minima on the potential energy surface. The parameter $\tau$ is arbitrary. 


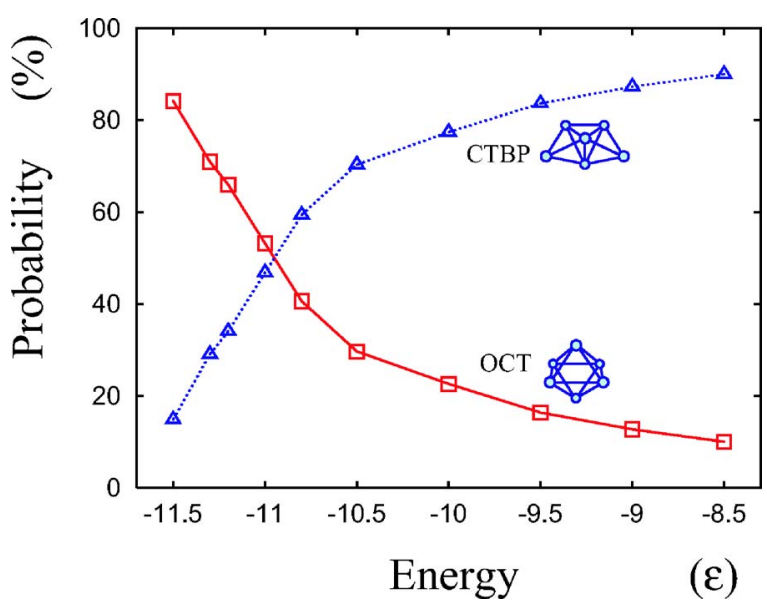

FIG. 2. (Color online) Energy dependence of the residence probabilities of the $\mathrm{M}_{6}$ cluster in the two isomers. Open squares represent the residence probability for the OCT isomer, while the open triangles represent the residence probability for the CTBP isomer.

With this method, every point along a classical trajectory is attributed to one of the two isomers except for the saddle points.

\section{B. Switching of structural preference}

We present here numerical results on the residence times of the $\mathrm{M}_{6}$ cluster in the two different isomer wells, which are relevant to the main subject of this paper. Figure 2 shows the energy dependence of the percentages of the total residence times in the two isomer wells obtained through a long-time simulation. These are essentially the probabilities of finding the system in the respective configurations. In the low energy range, the $\mathrm{M}_{6}$ cluster spends more time in the OCT isomer than in the CTBP isomer. This is understandable from the viewpoint of the potential energy topography since the potential well of the OCT isomer is much deeper than that of CTBP, as shown in Fig. 1. However, as energy increases, the structural preference of the cluster switches dramatically, and the system prefers to be in the CTBP isomer than in the OCT. In the high energy range, the potential energy curve in Fig. 1 is obviously not sufficient for explaining residence probabilities.

From the point of view of the statistical reaction rate theory, the residence times in the respective isomers should be attributed to the densities of states (phase space volumes) of the respective isomers. In this respect, the result of Fig. 2 indicates that the density of states of the CTBP isomer increases much more steeply than that of the OCT isomer as the total energy increases. Amar and Berry ${ }^{56}$ proposed a statistical argument to account for this type of inversion of residence times observed in the $\mathrm{Ar}_{7}$ cluster after taking the number of permutational isomers into consideration. The authors of Refs. 57 and 58 directly computed the densities of states for the isomers of the $\mathrm{Ar}_{7}$ cluster by Monte Carlo methods and compared them with the molecular dynamics in the respective isomers. Although statistical approaches give correct estimates for the residence times as long as the dynamics is sufficiently ergodic, an accurate computation of the density of states is often a hard task, especially for large sys- tems. In addition, statistical approaches do not clarify the short term, dynamical origin of the intriguing transitions. It is also of some concern that Monte Carlo methods often disregard the intrinsic non-Euclidean nature of the molecular internal space, which may induce a non-negligible error in the internal density of states.

The methods of this paper are dynamical ones, which are different from (but should be complementary to) statistical approaches. We first reduce the dimensionality of the dynamics using collective variables, which are the three gyration radii. Then the reaction dynamics are investigated within the framework of these reduced dynamics. The switching of the dominant conformation of the cluster in Fig. 2 will be accounted for (in Sec. IV) as a consequence of a general tendency inherent in the dynamics of polyatomic molecules: Molecules generally prefer to be in distorted (elongated) mass distributions (as in the CTBP isomer) instead of being in spherical mass distributions (as in the OCT isomer) in the high energy range.

\section{Gyration radii as collective variables}

We introduce here the gyration radii, our collective variables, and characterize the isomerization dynamics of the $\mathrm{M}_{6}$ cluster in the three-dimensional space of these radii. For generality, we first assume a general $n$-atom molecule whose constituent atoms have masses $\left\{m_{i} \mid i=1, \ldots, n\right\}$.

After eliminating the overall translational degrees of freedom of the molecule via the mass-weighted Jacobi vectors,

$$
\boldsymbol{\rho}_{s i}=\sqrt{\mu_{i}}\left(\frac{\sum_{k=1}^{i} m_{k} \boldsymbol{r}_{s k}}{\sum_{k=1}^{i} m_{k}}-\boldsymbol{r}_{s(i+1)}\right) \quad(i=1, \ldots, n-1),
$$

where $\mu_{i}$ are the reduced masses defined as

$$
\mu_{i}=\frac{m_{i+1} \sum_{k=1}^{i} m_{k}}{\sum_{k=1}^{i+1} m_{k}} \quad(i=1, \ldots, n-1),
$$

the singular-value decomposition theorem ${ }^{59}$ can be applied to decompose the $3 \times(n-1)$-dimensional matrix $\mathrm{W}_{s}$ of Jacobi vectors defined as

$$
\mathrm{W}_{s}=\left(\begin{array}{lll}
\boldsymbol{\rho}_{s 1} & \cdots & \boldsymbol{\rho}_{s(n-1)}
\end{array}\right)
$$

into the product of the three matrices,

$$
\mathrm{W}_{s}=\mathrm{RNU}^{T},
$$

where

$$
\begin{aligned}
& \mathbf{R}=\left(\boldsymbol{e}_{1} \boldsymbol{e}_{2} \boldsymbol{e}_{3}\right), \\
& \mathrm{N}=\left(\begin{array}{ccc|ccc}
a_{1} & 0 & 0 & 0 & \cdots & 0 \\
0 & a_{2} & 0 & 0 & \cdots & 0 \\
0 & 0 & a_{3} & 0 & \cdots & 0
\end{array}\right) \equiv(\mathrm{A} \mid \mathrm{O}), \\
& \mathrm{U}=\left(\begin{array}{lllll}
\boldsymbol{u}_{1} & \boldsymbol{u}_{2} & \boldsymbol{u}_{3} \mid \boldsymbol{u}_{4} & \cdots & \boldsymbol{u}_{n-1}
\end{array}\right) \equiv(\mathrm{P} \mid \mathrm{Q}) .
\end{aligned}
$$

The symbol $T$ on the matrix $U$ in Eq. (6) indicates the matrix transpose. The matrix $R$ is a $3 \times 3$ orthogonal matrix [SO(3)], whose column vectors, $\boldsymbol{e}_{1}, \boldsymbol{e}_{2}, \boldsymbol{e}_{3}$, are proper orthogonal and normalized. The matrix $\mathrm{N}$ is a $3 \times(n-1)$ diag- 
onal matrix, which is split up into the left $3 \times 3$ diagonal matrix $\mathrm{A}$ and the right $3 \times(n-4)$ zero matrix $\mathrm{O}$ for later use in Sec. III. The diagonal elements (singular values), $a_{1}, a_{2}, a_{3}$, are the gyration radii, which are the key quantities of this study. Although there are many alternative definitions for the Jacobi vectors, gyration radii are independent of the choice of Jacobi vectors. They are non-negative and ordered according to

$$
a_{1} \geqslant a_{2} \geqslant a_{3} \geqslant 0 \text {. }
$$

The matrix $U$ is an $(n-1) \times(n-1)$ proper orthogonal matrix $[\mathrm{SO}(n-1)]$, whose column vectors, $\boldsymbol{u}_{1}, \ldots, \boldsymbol{u}_{n-1}$, are orthogonal and normalized. The matrix $U$ is split up into the left $(n-1) \times 3$ matrix $\mathrm{P}$ and the right $(n-1) \times(n-4)$ matrix $\mathrm{Q}$ for later convenience. The relationships among the vectors and the singular values $\left\{\boldsymbol{e}_{i}\right\},\left\{\boldsymbol{u}_{i}\right\},\left\{a_{i}\right\}(i=1,2,3)$ in Eqs. (7)-(9) are summarized in the Appendix. The following are a few highlights.

The three gyration radii are related to the principal moments of inertia, $M_{1}, M_{2}$, and $M_{3}\left(M_{3} \geqslant M_{2} \geqslant M_{1} \geqslant 0\right)$, by

$$
M_{1}=a_{2}^{2}+a_{3}^{2}, \quad M_{2}=a_{3}^{2}+a_{1}^{2}, \quad M_{3}=a_{1}^{2}+a_{2}^{2} .
$$

The matrix $R$ coincides with the principal-axis frame. In the principal-axis hyperspherical coordinates, details of which are presented in the next section, the principal-axis frame $R$ specifies the instantaneous orientation of the system, while $\mathrm{N}$ and $U$ determine the internal structure (size and symmetry) of the system. The gyration radii, $a_{1}, a_{2}, a_{3}$, measure the mass-weighted length of the system along the respective principal axes. They essentially characterize the mass distribution.

The values of the gyration radii of the $\mathrm{M}_{6}$ cluster at the potential minimum of the OCT isomer are $\left(a_{1}, a_{2}, a_{3}\right)$ $=(5.97,5.97,5.97)$, while those of the CTBP isomer are $\left(a_{1}, a_{2}, a_{3}\right)=(8.19,5.89,4.27)$. The coincidence of the three gyration radii at the OCT structure indicates that it has isotropic mass distribution (spherical top). The CTBP structure has an asymmetric mass distribution.

Figure 3(a) shows a typical time evolution of the three gyration radii of the $\mathrm{M}_{6}$ cluster at total energy $E=-11.0 \varepsilon$. In the time period of Fig. 3(a), the system first transited from the OCT isomer to the CTBP isomer. After a while it returned back to the OCT isomer. The isomerization reaction is clearly marked by the change of the three gyration radii. In each isomer, the three gyration radii take values close to those at the corresponding potential minimum. Figure 3(b) shows the same trajectory as in Fig. 3(a) projected onto the three-dimensional space of gyration radii, which captures the changes of mass distribution of the system. In Fig. 3(b), the two regions corresponding to the OCT isomer and the CTBP isomer are distinguished. Both of the two regions are small when the total energy is low. As the total energy increases, the two regions become larger. The essential fact is that certain regions of the three-dimensional space of gyration radii and certain isomer wells of the cluster have good one-to-one correspondence. Therefore, the transition of the trajectory in the three-dimensional space of gyration radii from one region to the other is indicative of a structural change of the full system. This is the most vital condition for the three
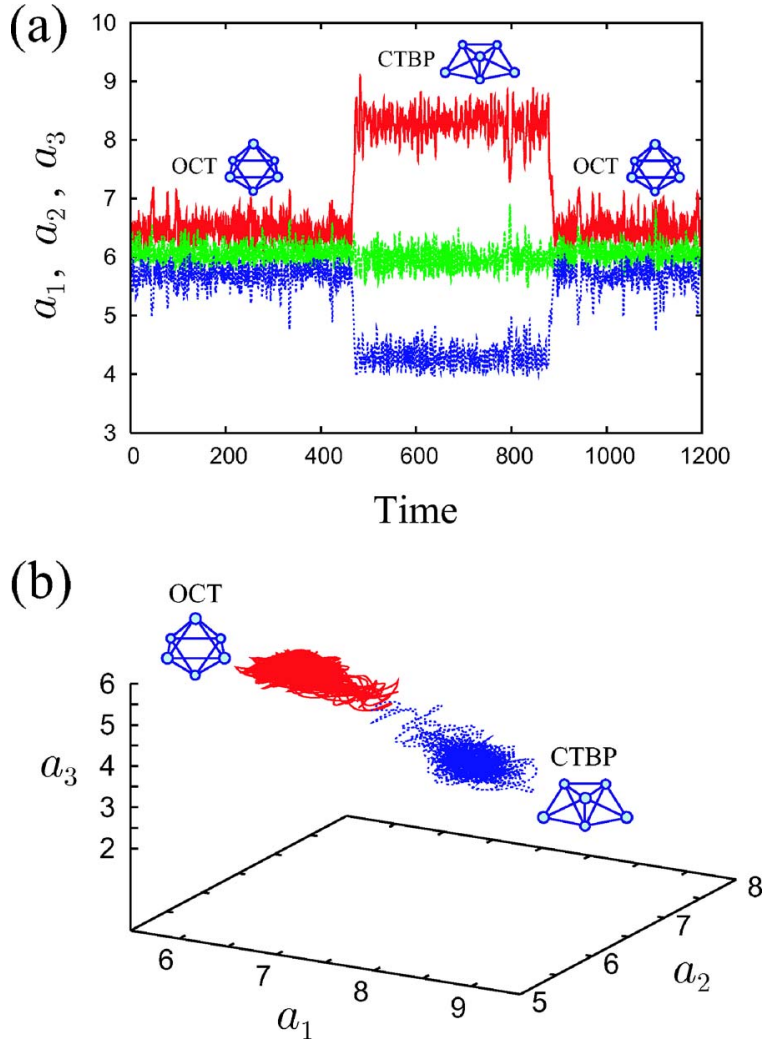

FIG. 3. (Color online) (a) Typical time evolution of the three gyration radii $a_{1}, a_{2}$, and $a_{3}\left(a_{1} \geqslant a_{2} \geqslant a_{3}\right)$ of the $\mathrm{M}_{6}$ cluster at total energy $E=-11.0 \varepsilon$. The cluster quenches to the OCT isomer from $t=0$ to $t=468$ and from $t=884$ to $t=1200$, while the cluster is quenched to the CTBP isomer from $t=468$ to $t=884$. (b) The same trajectory as in (a) projected onto the threedimensional space of gyration radii. The two trapping regions correspond to the two isomers, OCT (solid line) and CTBP (dashed line).

gyration radii to be good collective variables. In general, gyration radii can characterize structural transitions of a molecule in this way as long as the system changes its mass distribution significantly through the transitions.

The number of total internal degrees of freedom of the $\mathrm{M}_{6}$ cluster is 12 , which is the total number of degrees of freedom, 18, minus the three rotational and the three translational degrees of freedom. Therefore nine other internal degrees of freedom are "hidden" behind the dynamics in Fig. 3(b). If these hidden degrees of freedom can be regarded as bath modes, one can think of a closed dynamical system in terms of only the three gyration radii. If this is the case, one will be able to extract essential information about the structural transition of the full system from the low-dimensional dynamics of gyration radii. These are the main issues of this paper in the following sections, where we will finally extract an effective barrier structure that separates the different isomer regions as in Fig. 3(b). This barrier structure will in turn explain the true dynamical stability of the respective isomers.

\section{CHARACTERIZATION OF A KINEMATIC FORCE IN THE THREE-DIMENSIONAL SPACE OF GYRATION RADII}

The main subject of this section is to characterize the kinematic force arising in the three-dimensional space of gyration radii of an isolated $n$-atom molecule. We derive clas- 
sical equations of motion for the gyration radii using the principal-axis hyperspherical coordinates. We focus mainly on the effects that originate from the internal kinetic energy or equivalently from the internal space geometry in this section.

\section{A. Kinetic energy of an $n$-atom molecule with respect to a body frame}

We note here the general expression for the total angular momentum and that for the total kinetic energy in terms of the quantities with respect to a body (body-fixed) frame $\mathrm{R} \in \mathrm{SO}(3)$. In the next subsection, we identify the body frame $R$ as the principal-axis frame introduced in Eqs. (6) and (7). The goal of these two subsections is to obtain a concise and physically appealing kinetic energy expression such as Eqs. (33) and (35) below.

Since the Jacobi vectors with respect to the body frame $\left\{\boldsymbol{\rho}_{i} \mid i=1, \ldots, n-1\right\}$ are related to the Jacobi vectors with respect to the space-fixed frame $\left\{\boldsymbol{\rho}_{s i} \mid i=1, \ldots, n-1\right\}$ by $\boldsymbol{\rho}_{s i}$ $=\mathbf{R} \boldsymbol{\rho}_{i}$, the time-derivative of $\boldsymbol{\rho}_{s i}$ can be expressed as

$$
\dot{\boldsymbol{\rho}}_{s i}=\dot{\mathrm{R}} \boldsymbol{\rho}_{i}+\mathrm{R} \dot{\boldsymbol{\rho}}_{i}
$$

Similarly, the total angular momentum with respect to the space-fixed frame $\boldsymbol{L}_{s}$ and that with respect to the body frame $\boldsymbol{L}$ are related by $\boldsymbol{L}_{s}=\mathrm{R} \boldsymbol{L}$. Since

$$
\boldsymbol{L}_{s}=\sum_{i=1}^{n-1} \boldsymbol{\rho}_{s i} \times \dot{\boldsymbol{\rho}}_{s i}
$$

$\boldsymbol{L}$ can be expressed as

$$
\boldsymbol{L}=\mathrm{R}^{T} \sum_{i=1}^{n-1} \boldsymbol{\rho}_{s i} \times \dot{\boldsymbol{\rho}}_{s i}=\mathrm{M} \boldsymbol{\Omega}+\sum_{i=1}^{n-1} \boldsymbol{\rho}_{i} \times \dot{\boldsymbol{\rho}}_{i},
$$

where Eq. (12) is used in the second equality. The matrix M in Eq. (14) is the moment of inertia tensor with respect to the body frame, whose components are defined by

$$
M_{\alpha \beta}=\sum_{i=1}^{n-1}\left[\left(\boldsymbol{\rho}_{i} \cdot \boldsymbol{\rho}_{i}\right) \delta_{\alpha \beta}-\rho_{i \alpha} \rho_{i \beta}\right],
$$

where $\alpha$ and $\beta(\alpha, \beta=1,2,3)$ represent the axes of the body frame and $\delta_{\alpha \beta}$ is the Kronecker delta. The vector $\boldsymbol{\Omega}$ $\equiv\left(\Omega_{1}, \Omega_{2}, \Omega_{3}\right)^{T}$ in Eq. (14) is the angular velocity with respect to the body frame whose components are defined through the antisymmetric matrix,

$$
R^{T} \dot{R} \equiv\left(\begin{array}{ccc}
0 & -\Omega_{3} & \Omega_{2} \\
\Omega_{3} & 0 & -\Omega_{1} \\
-\Omega_{2} & \Omega_{1} & 0
\end{array}\right) .
$$

The kinetic energy of the system in a space-fixed frame is given by

$$
K=\frac{1}{2} \sum_{i=1}^{n} m_{i}\left(\dot{\boldsymbol{r}}_{s i} \cdot \dot{\boldsymbol{r}}_{s i}\right)=\frac{1}{2} \sum_{i=1}^{n-1} \dot{\boldsymbol{\rho}}_{s i} \cdot \dot{\boldsymbol{\rho}}_{s i} .
$$

After applying Eq. (12) to Eq. (17), it can be expressed in terms of the body-frame quantities as

$$
K=\frac{1}{2} \boldsymbol{\Omega}^{T} \mathrm{M} \boldsymbol{\Omega}+\boldsymbol{\Omega}^{T} \sum_{i=1}^{n-1} \boldsymbol{\rho}_{i} \times \dot{\boldsymbol{\rho}}_{i}+\frac{1}{2} \sum_{i=1}^{n-1} \dot{\boldsymbol{\rho}}_{i} \cdot \dot{\boldsymbol{\rho}}_{i},
$$

where the second term on the right hand side of Eq. (18) is often called the Coriolis coupling term.

\section{B. The kinetic energy in the principal-axis hyperspherical coordinates}

We reformulate here the kinetic energy of Eq. (18) using the principal-axis frame, gyration radii, and internal quasivelocities. After identifying the body frame $R$ in the previous subsection with the principal-axis frame $R$ introduced in Eqs. (6) and (7), the angular velocity vector $\boldsymbol{\Omega}$, the moment of inertia tensor M, and the Jacobi vectors $\left\{\boldsymbol{\rho}_{i}\right\}$ in Eq. (18) are all regarded as the ones with respect to the principal-axis frame.

Now the second term of Eq. (18) can be reformulated as follows. Based on Eq. (6), the Jacobi vectors $\left\{\boldsymbol{\rho}_{i}\right\}$ with respect to the principal-axis frame are expressed collectively in a matrix form as

$$
\left(\begin{array}{lll}
\boldsymbol{\rho}_{1} & \cdots & \boldsymbol{\rho}_{n-1}
\end{array}\right)=\mathrm{NU}^{T} \equiv \mathrm{W} .
$$

where the $3 \times(n-1)$ matrix $\mathrm{W}$ is a body-frame counterpart of $\mathbf{W}_{s}$ of Eq. (5). By using $\mathbf{W}$, the components of $\sum_{i=1}^{n-1} \boldsymbol{\rho}_{i}$ $\times \dot{\boldsymbol{\rho}}_{i} \equiv\left(\xi_{1}, \xi_{2}, \xi_{3}\right)^{T}$ are given by

$$
\left(\begin{array}{ccc}
0 & -\xi_{3} & \xi_{2} \\
\xi_{3} & 0 & -\xi_{1} \\
-\xi_{2} & \xi_{1} & 0
\end{array}\right)=\dot{\mathrm{W}} \mathrm{W}^{T}-\mathrm{WW}^{T}=-2 \mathrm{AP} \mathrm{P}^{T} \dot{\mathrm{P} A}
$$

where $A$ and $P$ are the submatrices defined in Eq. (8) and in Eq. (9). After introducing the quasivelocity components,

$$
\dot{\boldsymbol{u}}_{i} \cdot \boldsymbol{u}_{j}=-\boldsymbol{u}_{i} \cdot \dot{\boldsymbol{u}}_{j} \equiv \omega_{i j} \quad(i, j=1,2,3, i \neq j)
$$

the antisymmetric matrix $\mathrm{P}^{T} \dot{\mathrm{P}}$ can be expressed as

$$
\mathrm{P}^{T} \dot{\mathrm{P}}=\left(\begin{array}{ccc}
0 & -\omega_{12} & \omega_{31} \\
\omega_{12} & 0 & -\omega_{23} \\
-\omega_{31} & \omega_{23} & 0
\end{array}\right)
$$

By substituting Eq. (22) into Eq. (20) and comparing the leftmost part with the rightmost part of Eq. (20), we obtain

$$
\xi_{1}=-2 a_{2} a_{3} \omega_{23}, \quad \xi_{2}=-2 a_{3} a_{1} \omega_{31}, \quad \xi_{3}=-2 a_{1} a_{2} \omega_{12} .
$$

These formulas allow us to express the second term on the right hand side of Eq. (18) finally as 


$$
\begin{aligned}
\boldsymbol{\Omega}^{T} \sum_{i=1}^{n-1} \boldsymbol{\rho}_{i} \times \dot{\boldsymbol{\rho}}_{i}= & -2 a_{2} a_{3} \Omega_{1} \omega_{23}-2 a_{3} a_{1} \Omega_{2} \omega_{31} \\
& -2 a_{1} a_{2} \Omega_{3} \omega_{12} \\
& =\boldsymbol{\Omega}^{T} \mathrm{~B} \boldsymbol{\omega}
\end{aligned}
$$

where the following two matrices are introduced for notational compactness:

$$
\mathrm{B}=\left(\begin{array}{ccc}
-2 a_{2} a_{3} & 0 & 0 \\
0 & -2 a_{3} a_{1} & 0 \\
0 & 0 & -2 a_{1} a_{2}
\end{array}\right), \quad \boldsymbol{\omega}=\left(\begin{array}{c}
\omega_{23} \\
\omega_{31} \\
\omega_{12}
\end{array}\right)
$$

Similarly, the third term in the right hand side of Eq. (18) can also be reformulated using the matrix W of Eq. (19) as

$$
\begin{aligned}
\frac{1}{2} \sum_{i=1}^{n-1} \dot{\boldsymbol{\rho}}_{i} \cdot \dot{\boldsymbol{\rho}}_{i}= & \frac{1}{2} \operatorname{Tr}\left(\dot{\mathrm{W}} \dot{\mathrm{W}}^{T}\right) \\
= & \frac{1}{2} \operatorname{Tr}\left(\dot{\mathrm{A}} \dot{\mathrm{A}}^{T}\right)+\frac{1}{2} \operatorname{Tr}\left(\mathrm{A} \dot{\mathrm{P}}^{T} \mathrm{PP}{ }^{T} \dot{\mathrm{P}} \mathrm{A}\right) \\
& +\frac{1}{2} \operatorname{Tr}\left(\dot{\mathrm{AP}}^{T} \mathrm{Q} \mathrm{Q}^{T} \dot{\mathrm{PA}}\right),
\end{aligned}
$$

where $\operatorname{Tr}(\cdot)$ represents the trace of a matrix and where $A, P$, and $Q$ are the matrices defined in Eq. (8) and in Eq. (9). After introducing the quasivelocity components,

$$
\dot{\boldsymbol{u}}_{i} \cdot \boldsymbol{u}_{j}=-\boldsymbol{u}_{i} \cdot \dot{\boldsymbol{u}}_{j} \equiv \gamma_{i j} \quad(i=1,2,3, j=4, \ldots, n-1),
$$

the $(n-4) \times 3$ matrix $\mathrm{Q}^{T} \dot{\mathrm{P}}$ in Eq. (28) can be expressed as

$$
\mathbf{Q}^{T} \dot{\mathrm{P}}=\left(\begin{array}{ccc}
\gamma_{14} & \gamma_{24} & \gamma_{34} \\
\vdots & \vdots & \vdots \\
\gamma_{1(n-1)} & \gamma_{2(n-1)} & \gamma_{3(n-1)}
\end{array}\right) \equiv\left(\begin{array}{lll}
\gamma_{1} & \gamma_{2} & \gamma_{3}
\end{array}\right)
$$

where we have introduced the $(n-4)$-dimensional column vectors $\boldsymbol{\gamma}_{i}(i=1,2,3)$. By applying Eqs. (22) and (30) to Eq. (28), we obtain the final expression for the third term of Eq. (18) as

$$
\begin{aligned}
\frac{1}{2} \sum_{i=1}^{n-1} \dot{\boldsymbol{\rho}}_{i} \cdot \dot{\boldsymbol{\rho}}_{i}= & \frac{1}{2}\left(\dot{a}_{1}^{2}+\dot{a}_{2}^{2}+\dot{a}_{3}^{2}\right)+\frac{1}{2}\left(M_{1} \omega_{23}^{2}+M_{2} \omega_{31}^{2}+M_{3} \omega_{12}^{2}\right) \\
& +\frac{1}{2} a_{1}^{2} \sum_{k=4}^{n-1} \gamma_{1 k}^{2}+\frac{1}{2} a_{2}^{2} \sum_{k=4}^{n-1} \gamma_{2 k}^{2}+\frac{1}{2} a_{3}^{2} \sum_{k=4}^{n-1} \gamma_{3 k}^{2} \\
= & \frac{1}{2} \dot{\boldsymbol{a}} \cdot \dot{\boldsymbol{a}}+\frac{1}{2} \boldsymbol{\omega}^{T} \mathbf{M} \boldsymbol{\omega}+\frac{1}{2} \sum_{i=1}^{3} \boldsymbol{\gamma}_{i}^{T} a_{i}^{2} \boldsymbol{\gamma}_{i},
\end{aligned}
$$

where $\boldsymbol{a} \equiv\left(a_{1}, a_{2}, a_{3}\right)^{T}$, and $\mathrm{M}$ is the moment of inertia tensor with respect to the principal-axis frame [see Eq. (A4) in the Appendix].

Substituting Eqs. (25) and (32) into Eq. (18), we obtain the desired general expression for the total kinetic energy of an $n$-atom molecule as

$$
K=\frac{1}{2} \boldsymbol{\Omega}^{T} \mathrm{M} \boldsymbol{\Omega}+\boldsymbol{\Omega}^{T} \mathrm{~B} \boldsymbol{\omega}+\frac{1}{2} \dot{\boldsymbol{a}} \cdot \dot{\boldsymbol{a}}+\frac{1}{2} \boldsymbol{\omega}^{T} \mathrm{M} \boldsymbol{\omega}+\frac{1}{2} \sum_{i=1}^{3} \boldsymbol{\gamma}_{i}^{T} a_{i}^{2} \boldsymbol{\gamma}_{i},
$$

which is essentially the same as the one given by Eckart ${ }^{28}$ and by Chapuisat et al. ${ }^{29}$ Introduction of the quasivelocities, $\boldsymbol{\omega}$ (gyration quasivelocity) and $\boldsymbol{\gamma}_{i}$ (internal quasivelocity), has been crucial in making this a compact expression; in it, the $(3 n-6)$ internal degrees of freedom are represented by the three gyration radii and the $(3 n-9)$ quasivelocity components. These quasivelocities are essentially the angular velocities of kinematic (or democratic) rotations, which are continuous shape changes associated with the permutations (relabelings) among the constituent atoms. ${ }^{31-37}$ They should be distinguished from the angular velocity of the principalaxis frame $\boldsymbol{\Omega}$.

\section{Equations of motion for gyration radii under the conditions of vanishing total angular momentum}

We next investigate the equations of motion for the three gyration radii, $a_{1}, a_{2}$, and $a_{3}$, under the conditions of zero total angular momentum.

Since the total angular momentum with respect to the body frame is given by

$$
\boldsymbol{L}=\mathrm{M} \boldsymbol{\Omega}+\mathrm{B} \boldsymbol{\omega},
$$

after using Eqs. (14) and (25), the total kinetic energy can be expressed as follows after applying Eq. (34) to Eq. (33):

$$
K=\frac{1}{2} \boldsymbol{L}^{T} \mathrm{M}^{-1} \boldsymbol{L}+\frac{1}{2} \dot{\boldsymbol{a}} \cdot \dot{\boldsymbol{a}}+\frac{1}{2} \boldsymbol{\omega}^{T} \tilde{\mathrm{M}} \boldsymbol{\omega}+\frac{1}{2} \sum_{i=1}^{3} \boldsymbol{\gamma}_{i}^{T} a_{i}^{2} \boldsymbol{\gamma}_{i},
$$

where we have introduced a diagonal matrix,

$$
\tilde{\mathrm{M}} \equiv \mathrm{M}-\mathrm{BM}^{-1} \mathrm{~B}=\left(\begin{array}{ccc}
\left(a_{2}^{2}-a_{3}^{2}\right)^{2} /\left(a_{2}^{2}+a_{3}^{2}\right) & 0 & 0 \\
0 & \left(a_{3}^{2}-a_{1}^{2}\right)^{2} /\left(a_{3}^{2}+a_{1}^{2}\right) & 0 \\
0 & 0 & \left(a_{1}^{2}-a_{2}^{2}\right)^{2} /\left(a_{1}^{2}+a_{2}^{2}\right)
\end{array}\right)
$$


The expression of Eq. (35) is important from the gaugetheoretical point of view: ${ }^{27}$ Each term of Eq. (35) is independent of the choice of body frame, while the kinetic energy expression of Eq. (33) is specific to the principal-axis choice of body frame. By applying the zero angular momentum condition, $\boldsymbol{L}=\mathbf{0}$, to Eq. (35), we obtain the purely internal kinetic energy,

$$
\begin{aligned}
K= & \frac{1}{2} \dot{\boldsymbol{a}} \cdot \dot{\boldsymbol{a}}+\frac{1}{2} \boldsymbol{\omega}^{T} \tilde{\mathrm{M}} \boldsymbol{\omega}+\frac{1}{2} \sum_{i=1}^{3} \gamma_{i}^{T} a_{i}^{2} \boldsymbol{\gamma}_{i}, \\
& =\frac{1}{2}\left(\dot{a}_{1}^{2}+\dot{a}_{2}^{2}+\dot{a}_{3}^{2}\right)+\frac{\left(a_{1}^{2}-a_{2}^{2}\right)^{2}}{2\left(a_{1}^{2}+a_{2}^{2}\right)} \omega_{12}^{2}+\frac{\left(a_{2}^{2}-a_{3}^{2}\right)^{2}}{2\left(a_{2}^{2}+a_{3}^{2}\right)} \omega_{23}^{2} \\
& +\frac{\left(a_{3}^{2}-a_{1}^{2}\right)^{2}}{2\left(a_{3}^{2}+a_{1}^{2}\right)} \omega_{31}^{2}+\frac{1}{2} a_{1}^{2} \sum_{k=4}^{n-1} \gamma_{1 k}^{2}+\frac{1}{2} a_{2}^{2} \sum_{k=4}^{n-1} \gamma_{2 k}^{2} \\
& +\frac{1}{2} a_{3}^{2} \sum_{k=4}^{n-1} \gamma_{3 k}^{2} .
\end{aligned}
$$

At this point, it is important to note that the mass metric components for $\dot{a}_{1}^{2}, \dot{a}_{2}^{2}$, and $\dot{a}_{3}^{2}$ are all equal to unity. This means that the three-dimensional space of gyration radii such as Fig. 3(b) is Euclidean even though the $(3 n-6)$-dimensional full internal space is non-Euclidean. This will be of great advantage in the following discussions in reducing the full dynamics of a molecule to the threedimensional space of gyration radii.

By using the internal kinetic energy, Eq. (38), the Lagrangian for the $n$-atom molecule with vanishing total angular momentum is given by $\mathcal{L}=K-V$. Recall that for our study of isolated molecules the potential energy $V$ is a function only of the internal degrees of freedom. The Euler-Lagrange equations for the gyration radii can be written as

$$
\begin{aligned}
\ddot{a}_{1}= & \frac{a_{1}\left(a_{1}^{2}+3 a_{2}^{2}\right)\left(a_{1}^{2}-a_{2}^{2}\right)}{\left(a_{1}^{2}+a_{2}^{2}\right)^{2}} \omega_{12}^{2}+\frac{a_{1}\left(a_{1}^{2}+3 a_{3}^{2}\right)\left(a_{1}^{2}-a_{3}^{2}\right)}{\left(a_{1}^{2}+a_{3}^{2}\right)^{2}} \omega_{13}^{2} \\
& +a_{1} \sum_{k=4}^{n-1} \gamma_{1 k}^{2}-\frac{\partial V}{\partial a_{1}}, \\
\ddot{a}_{2}= & \frac{a_{2}\left(a_{2}^{2}+3 a_{1}^{2}\right)\left(a_{2}^{2}-a_{1}^{2}\right)}{\left(a_{2}^{2}+a_{1}^{2}\right)^{2}} \omega_{21}^{2}+\frac{a_{2}\left(a_{2}^{2}+3 a_{3}^{2}\right)\left(a_{2}^{2}-a_{3}^{2}\right)}{\left(a_{2}^{2}+a_{3}^{2}\right)^{2}} \omega_{23}^{2} \\
& +a_{2} \sum_{k=4}^{n-1} \gamma_{2 k}^{2}-\frac{\partial V}{\partial a_{2}}, \\
\ddot{a}_{3}= & \frac{a_{3}\left(a_{3}^{2}+3 a_{2}^{2}\right)\left(a_{3}^{2}-a_{2}^{2}\right)}{\left(a_{3}^{2}+a_{2}^{2}\right)^{2}} \omega_{32}^{2}+\frac{a_{3}\left(a_{3}^{2}+3 a_{1}^{2}\right)\left(a_{3}^{2}-a_{1}^{2}\right)}{\left(a_{3}^{2}+a_{1}^{2}\right)^{2}} \omega_{31}^{2} \\
& +a_{3} \sum_{k=4}^{n-1} \gamma_{3 k}^{2}-\frac{\partial V}{\partial a_{3}} .
\end{aligned}
$$

The left hand sides of these equations are the components of acceleration in the three-dimensional space of gyration radii. The right hand sides are physically the forces in this space. While the fourth terms on the right hand sides of Eqs. (39)-(41) represent the force that originates from the poten- tial energy function, the first three terms on the right hand sides of these equations represent a kinematic force that originates from the dynamical coupling of gyration radii with the kinematic-rotation modes. All of these kinematic force terms are quadratic in the quasivelocity components, $\omega_{i j}$ and $\gamma_{i k}$. Therefore, these terms essentially represent the internal centrifugal force arising from the kinematic rotations. It has been shown that the internal centrifugal terms proportional to $\omega_{i j}^{2}$ arise in the three- and four-atom dynamics. ${ }^{41}$ But the internal centrifugal force terms proportional to $\gamma_{i k}^{2}$ are intrinsic to systems with more than four-atom systems in the threedimensional physical space. As the number of atoms increases, the number of the terms proportional to $\gamma_{i k}^{2}$ increases.

While the force arising from the potential function is dependent on the system, the internal centrifugal force terms in Eqs. (39)-(41) are common to the dynamics of general $n$-atom molecules. Therefore it is quite interesting to explore the essential properties of this kinematic force. The internal centrifugal force terms proportional to $\omega_{i j}^{2}$ have an effect of breaking the symmetry of mass distribution of the $n$-atom molecule. This property can be understood by noting the sign of the corresponding terms in Eqs. (39)-(41): Since $a_{1} \geqslant a_{2}$ $\geqslant a_{3} \geqslant 0$ holds by definition, the first and the second terms in Eq. (39) are always positive or zero. This means that these terms have a persistent effect of enlarging the largest gyration radius $a_{1}$. On the other hand, by similar arguments, the first and the second terms in Eq. (41) are always negative or zero. These terms will therefore persistently diminish the smallest gyration radius $a_{3}$. Finally, the first term of Eq. (40) is negative or zero and the second term of Eq. (40) is positive or zero. Therefore, the force can act both positively and negatively for $a_{2}$. Since the gyration radii are the measures of the mass-weighted size of the system along the principal axes, these properties of the internal centrifugal force indicate that a molecule is always forced to elongate in the most massive direction and to collapse in the least massive direction. Properties of the internal centrifugal force terms proportional to $\gamma_{i k}^{2}$ are different from those proportional to $\omega_{i j}^{2}$. The terms proportional to $\gamma_{i k}^{2}$ in Eqs. (39)-(41) are always positive. Therefore, these have an effect of inflating the molecule in all three principal-axis directions. It is expected that the inflating effect becomes greater as the number of atoms increases, since the number of the terms proportional to $\gamma_{i k}^{2}$ increases.

In the above characterization of the directionality of the internal centrifugal force, it is essential that they are all quadratic in $\omega_{i j}$ and $\gamma_{i k}$. Although these quasivelocities can generally be positive or negative, their squares are always nonnegative. This quadratic nature of the internal centrifugal force terms is crucial in their persistent directionality. The properties of the internal centrifugal force discussed above have similarities to those of the normal centrifugal force (in the presence of nonzero angular momentum). However the origins of the internal centrifugal force and the normal centrifugal force are different. The normal centrifugal force is associated with the non-zero total angular momentum and is thereby absent in the system with zero total angular momentum. On the other hand, the internal centrifugal force of this 
study is associated with the kinematic rotations and can arise even in the dynamics with zero angular momentum.

\section{Averaged field of internal centrifugal force and geodesics in molecular internal space}

In this paper, the three gyration radii $\left(a_{1}, a_{2}, a_{3}\right)$ are regarded as collective variables, as we discussed in Sec. II C, while all other internal degrees of freedom are regarded as bath modes. Therefore our basic strategy is to average out the quasivelocity components $\left\{\omega_{i j}\right\}$ and $\left\{\gamma_{i k}\right\}$ in Eqs. (39)-(41) to estimate the effective internal centrifugal force that gyration radii "feel" in their three-dimensional space such as in Fig. 3(b). Here, we present one possible way of doing this in a special case where there is no potential force and where we can focus on the essential properties of the internal centrifugal force.

Since the internal centrifugal force originates from the internal kinetic energy of Eq. (38) and not from the potential function, the effect of this kinematic force stands out in the dynamics determined only by the internal kinetic energy. These are physically the dynamics of geodesics in the $(3 n$ -6)-dimensional molecular internal space. ${ }^{27}$ Therefore we consider the following situation: Consider an ensemble of geodesics having random initial velocities and the same kinetic energy $K_{g}$, which is a constant of motion along each geodesic. Then the statistical tendency of the behavior of these geodesics projected on in the three-dimensional space of gyration radii can be characterized by an averaged field of the internal centrifugal force. This averaged force field is evaluated at each point of the space of gyration radii as $\left(\left\langle f_{\text {cent }, 1}\right\rangle,\left\langle f_{\text {cent }, 2}\right\rangle,\left\langle f_{\text {cent }, 3}\right\rangle\right)$, where

$$
\begin{aligned}
&\left\langle f_{\text {cent }, i}\right\rangle= \frac{a_{i}\left(a_{i}^{2}+3 a_{j}^{2}\right)\left(a_{i}^{2}-a_{j}^{2}\right)}{\left(a_{i}^{2}+a_{j}^{2}\right)^{2}}\left\langle\omega_{i j}^{2}\right\rangle \\
&+\frac{a_{i}\left(a_{i}^{2}+3 a_{k}^{2}\right)\left(a_{i}^{2}-a_{k}^{2}\right)}{\left(a_{i}^{2}+a_{k}^{2}\right)^{2}}\left\langle\omega_{i k}^{2}\right\rangle+a_{i} \sum_{l=4}^{n-1}\left\langle\gamma_{i l}^{2}\right\rangle \\
&(i, j, k=1,2,3, \quad i \neq j, \quad j \neq k, \quad k \neq i) .
\end{aligned}
$$

In Eq. (42), all the brackets $\langle\cdot\rangle$ represent an ensemble average over the geodesics at each point of the space of gyration radii $\left(a_{1}, a_{2}, a_{3}\right)$. Since the geodesics under consideration have random initial velocities, the kinetic energy $K_{g}$ should be equipartitioned on average among the terms in Eq. (38) at each point of the space of gyration radii. Under this situation, we obtain

$$
\begin{aligned}
& \frac{\left(a_{i}^{2}-a_{j}^{2}\right)^{2}}{2\left(a_{i}^{2}+a_{j}^{2}\right)}\left\langle\omega_{i j}^{2}\right\rangle=\frac{K_{g}}{3 n-6} \quad(i, j=1,2,3, \quad i \neq j), \\
& \frac{1}{2} a_{i}^{2}\left\langle\gamma_{i k}^{2}\right\rangle=\frac{K_{g}}{3 n-6} \quad(i=1,2,3, \quad k=4, \ldots, n-1) .
\end{aligned}
$$

Applying Eqs. (43) and (44) to Eq. (42), the averaged field of the internal centrifugal force is deduced as
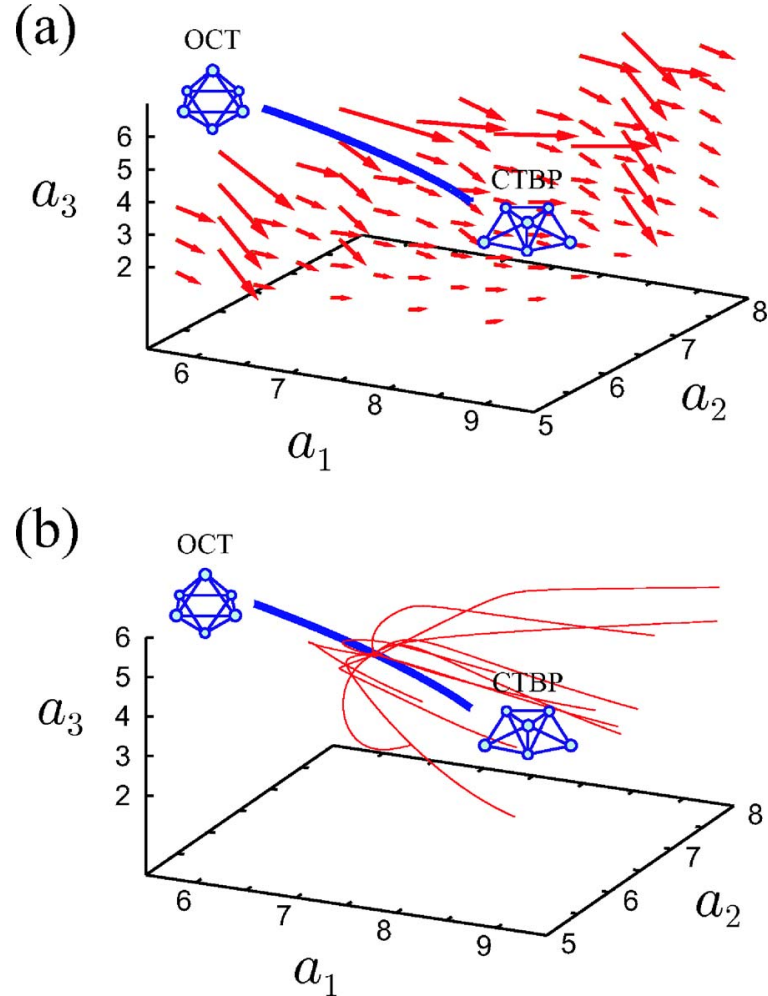

FIG. 4. (Color online) (a) Averaged field of internal centrifugal force in the space of gyration radii. The arrows represent the directionality and the magnitude of the averaged internal centrifugal force at respective points. The thick solid curve is the projected steepest descent path in the space of gyration radii for comparison. (b) Thin solid curves are the geodesics in the internal space projected onto the space of gyration radii. The geodesics start at the vicinity of the point corresponding to the saddle point of the system with random initial velocities.

$$
\begin{aligned}
& \left\langle f_{\text {cent }, 1}\right\rangle=\frac{2 K_{g}}{3 n-6}\left[\frac{a_{1}\left(a_{1}^{2}+3 a_{2}^{2}\right)}{a_{1}^{4}-a_{2}^{4}}+\frac{a_{1}\left(a_{1}^{2}+3 a_{3}^{2}\right)}{a_{1}^{4}-a_{3}^{4}}+\frac{n-4}{a_{1}}\right], \\
& \left\langle f_{\text {cent }, 2}\right\rangle=\frac{2 K_{g}}{3 n-6}\left[\frac{a_{2}\left(a_{2}^{2}+3 a_{1}^{2}\right)}{a_{2}^{4}-a_{1}^{4}}+\frac{a_{2}\left(a_{2}^{2}+3 a_{3}^{2}\right)}{a_{2}^{4}-a_{3}^{4}}+\frac{n-4}{a_{2}}\right],
\end{aligned}
$$

$$
\left\langle f_{\text {cent }, 3}\right\rangle=\frac{2 K_{g}}{3 n-6}\left[\frac{a_{3}\left(a_{3}^{2}+3 a_{2}^{2}\right)}{a_{3}^{4}-a_{2}^{4}}+\frac{a_{3}\left(a_{3}^{2}+3 a_{1}^{2}\right)}{a_{3}^{4}-a_{1}^{4}}+\frac{n-4}{a_{3}}\right] .
$$

Notice that the dependence on the quasivelocities in Eqs. (39)-(41) has been averaged out in Eqs. (45)-(47), and this force field is a function of the gyration radii $\left(a_{1}, a_{2}, a_{3}\right)$ only.

Figure 4(a) shows the averaged field of the internal centrifugal force of Eqs. (45)-(47) in the space of gyration radii for the six-atom system, $n=6$. The arrows represent the directionality and the magnitude of the force field at each point. In the figure, the projection of the steepest descent path connecting the saddle point and the two potential minima of the $\mathrm{M}_{6}$ cluster is also shown for comparison. The end points of the path correspond to the potential minima of the cluster as indicated. Notice the directionality of the internal centrifugal force as we discussed in Sec. III C: The ar- 
rows are directed in the positive direction for $a_{1}$ and in either direction for $a_{2}$ and $a_{3}$. As can be seen from Eqs. (45)-(47), the magnitude of the averaged internal centrifugal force tends to be large in the region where the two or all three of the gyration radii are close to each other. In fact, if two or three of the gyration radii are equal, which is the case at the minimum point of the OCT isomer, the averaged internal centrifugal force diverges to infinity. For this reason, arrows are not drawn in regions where two or three gyration radii are numerically very close to each other in Fig. 4(a).

In order to demonstrate the validity of the averaged force field, we numerically evolved an ensemble of geodesics in the internal space of six-atom system. Figure 4(b) shows an ensemble of geodesics with random initial velocities (and with constant energy) that start from the vicinity of the point which corresponds to the saddle point of the $M_{6}$ cluster. There is a clear correlation between the behavior of the geodesics and the direction of the averaged force field in the space of gyration radii. This clearly shows the actual significance of the internal centrifugal force that makes the system elongated and inflated. It is extremely important in view of the isomerization dynamics of our $\mathrm{M}_{6}$ cluster that the geodesics already have a strong tendency to bounce from the region of the OCT isomer and to evolve in the direction of the CTBP isomer. This is because the averaged field of the internal centrifugal force has the directionality to "push" the geodesics from the OCT region to the CTBP region. The geodesics finally go to infinity. This tendency of geodesics clearly shows that the mass distribution of the CTBP isomer is kinematically more preferred than that of the OCT isomer. Note again that these results are induced by the internal kinetic energy only. In the next section, we merge these kinematic effects with the effects of the potential energy function in the three-dimensional space of gyration radii.

\section{COMPETITION BETWEEN KINEMATIC FORCE AND POTENTIAL FORCE IN THE THREE-DIMENSIONAL SPACE OF GYRATION RADII}

In this section, we explore the mechanism of the structural transition dynamics of the $\mathrm{M}_{6}$ cluster in the threedimensional space of gyration radii. The competition between the kinematic force discussed in the previous section and the potential force is highlighted.

\section{A. Internal centrifugal force and potential force in the cluster dynamics}

We first investigate the time scales of the quantities in Eqs. (39)-(41) that are responsible for the dynamics of the gyration radii. Figure 5 shows the typical time evolution of (a) the gyration radii $a_{1}, a_{2}$, and $a_{3}$, (b) the squares of the gyration quasivelocities $\omega_{i j}^{2}$, (c) the squares of the internal quasivelocities, $\gamma_{i}^{2} \equiv \gamma_{i 4}^{2}+\gamma_{i 5}^{2}(i=1,2,3)$, and (d) the magnitude of the potential force and that of the internal centrifugal force acting on the gyration radii in the dynamics of
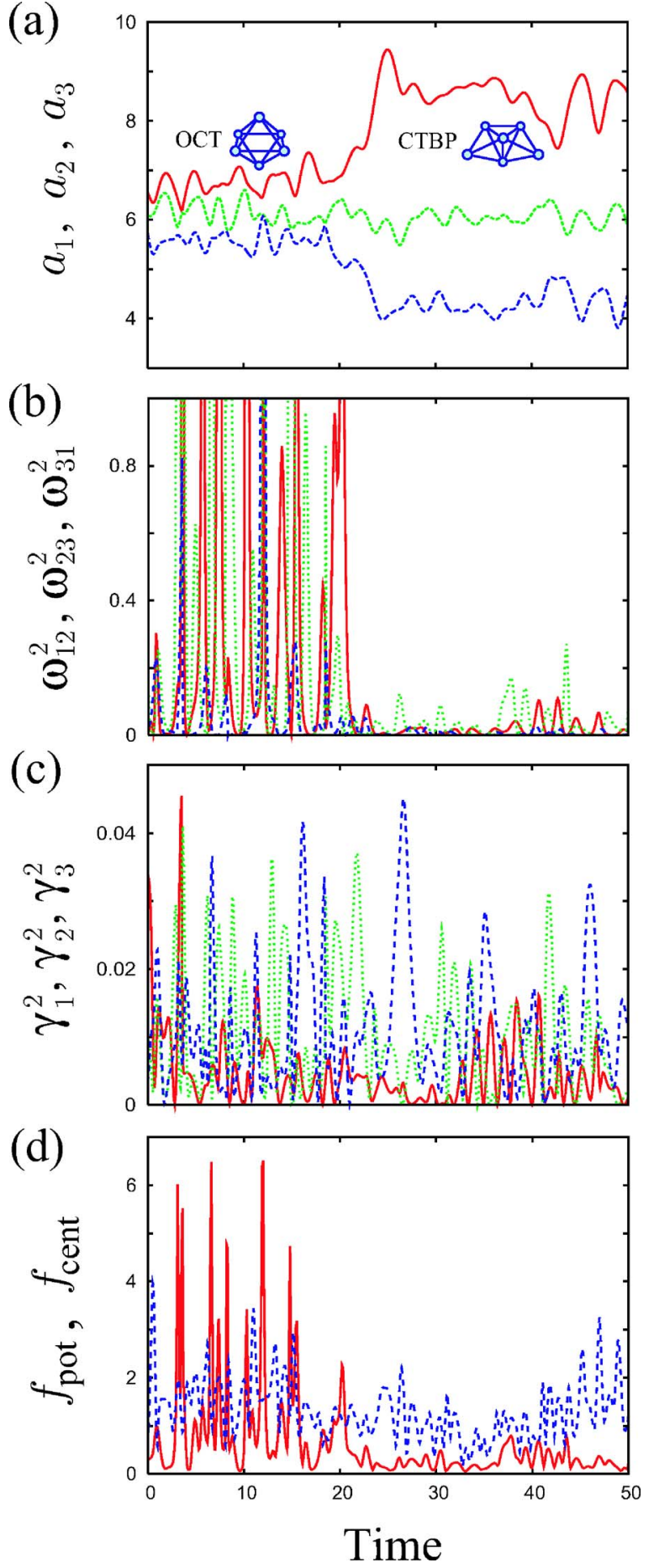

FIG. 5. (Color online) Typical time evolution of (a) the three gyration radii $a_{1}, a_{2}$, and $a_{3}$, (b) the squares of the gyration quasivelocities, $\omega_{12}^{2}, \omega_{23}^{2}, \omega_{31}^{2}$, (c) the squares of the internal quasivelocities, $\gamma_{1}^{2}, \gamma_{2}^{2}, \gamma_{3}^{2}$, and (d) magnitude of the potential force $f_{\text {pot }}$ (broken curve), and that of the internal centrifugal force $f_{\text {cent }}$ (solid curve) at total energy $E=-10.0 \varepsilon$. The system quenches to OCT from $t=0$ to $t=21.5$, and to CTBP from $t=21.5$ to $t=50$.

the $\mathrm{M}_{6}$ cluster at total energy $E=-10.0 \varepsilon$. The magnitude of the potential force is defined by

$$
f_{\mathrm{pot}}=\sqrt{\left(\frac{\partial V}{\partial a_{1}}\right)^{2}+\left(\frac{\partial V}{\partial a_{2}}\right)^{2}+\left(\frac{\partial V}{\partial a_{3}}\right)^{2}}
$$

while the magnitude of the internal centrifugal force is defined by 
(a)

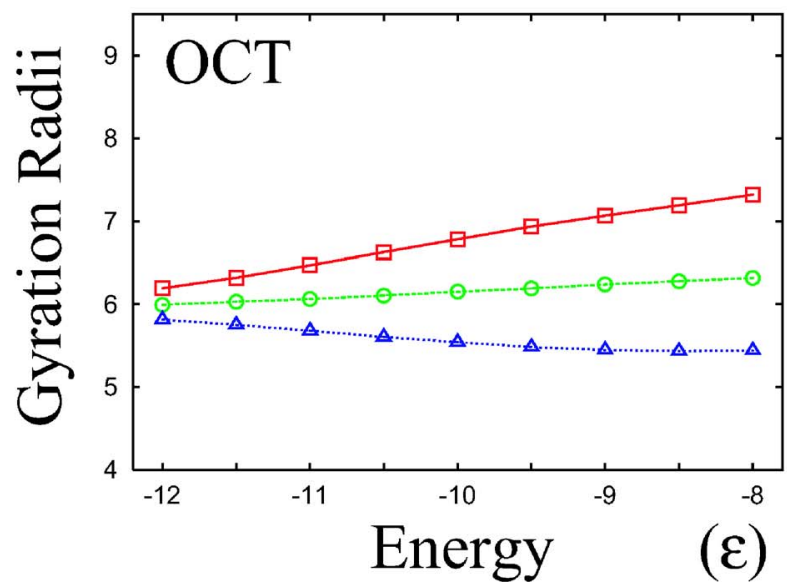

(b)

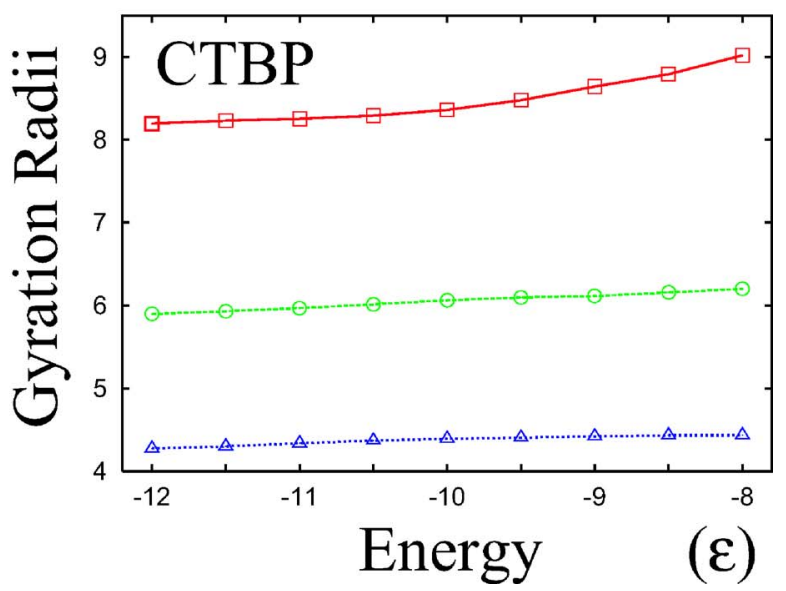

FIG. 6. (Color online) Energy dependence of the average values of the three gyration radii (a) for the OCT isomer and (b) for the CTBP isomer.

$$
f_{\text {cent }}=\sqrt{f_{\text {cent }, 1}^{2}+f_{\text {cent }, 2}^{2}+f_{\text {cent }, 3}^{2}}
$$

where each $f_{\text {cent }, i}$ is defined as the sum of the first, second, and third terms of each of Eqs. (39)-(41). Since the space of gyration radii is Euclidean, no non-Euclidean metric has to be taken into account in the definitions. During the time interval of Fig. 5, the cluster had undergone isomerization from the OCT isomer to the CTBP isomer once.

In Fig. 5, it can be seen that the three gyration radii, $a_{1}$, $a_{2}$, and $a_{3}$, change slowly and smoothly, in contrast to the internal quasivelocity components, $\omega_{i j}^{2}$ and $\gamma_{i}^{2}$, which oscillate rapidly and sharply. This result supports the ansatz that the gyration radii can be considered as slow variables while other internal modes behave like bath modes. The rapid oscillations of the quasivelocities induce the rapid oscillations of the internal centrifugal force as in Fig. 5(d), where the potential force also oscillates rapidly. One may consider these rapid oscillations as "masked" behind the slow dynamics of gyration radii. This result justifies our strategy of averaging the rapid oscillations of the forces in Eqs. (39)-(41) to deduce effective force fields that the gyration radii feel as will be shown below.

Figure 5 also reveals several other important facts in the internal motions of the cluster. First, the magnitude of the internal centrifugal force is as significant as that of the potential force [see Fig. 5(d)]. Therefore the effect of the internal centrifugal force is never negligible in the dynamics of gyration radii. Secondly, the quasivelocity components, $\omega_{12}^{2}$, $\omega_{23}^{2}, \omega_{31}^{2}$, and the magnitude of the internal centrifugal force in the OCT isomer are much larger than those in the CTBP isomer [see Figs. 5(b) and 5(d)]. The reason for these differences lies in the difference in the shapes of the two isomers themselves: Since the OCT conformation has an isotropic mass distribution, the values of the three gyration radii are close to each other when the system is close to this conformation [see Fig. 5(a)]. Therefore, the three diagonal components of $\tilde{M}$ of Eq. (36), which are essentially the effective moments of inertia for the kinematic rotations, are very small in the OCT conformation. As a result, in the OCT isomer, the components of the quasivelocity $\boldsymbol{\omega}$ of Eq. (38) can be very large inducing, in turn, the strong internal centrifugal force of Eqs. (39)-(41). On the other hand, in the CTBP isomer, values of the three gyration radii are separated and the components of $\tilde{M}$ are appreciable. Therefore the quasivelocity and the internal centrifugal force cannot be large in the CTBP isomer. In this way, the cluster geometry and the magnitude of the internal centrifugal force are highly correlated. The magnitude of the potential force does not appear significantly different in the two isomers [see Fig. 5(d)].

\section{B. Symmetry breaking effect of the internal centrifugal force}

We next investigate the competition between the internal centrifugal force and the potential force in the respective isomers of the $\mathrm{M}_{6}$ cluster.

As we discussed in Sec. III C, the internal centrifugal force induces a symmetry breaking effect [the first and the second terms in Eqs. (39)-(41)] and an inflating effect [the third terms in Eqs. (39)-(41)] on the mass distribution of the system. These effects often act counter to the effects of potential force. For example, in the OCT isomer of the $\mathrm{M}_{6}$ cluster, the potential force generally works to restore the symmetric (isotropic) and compact mass distribution at the potential minimum. However, the internal centrifugal force works against such a symmetric and compact mass distribution and prevents the system from getting close to the potential minimum of the OCT isomer. This effect of the internal centrifugal force has already been seen in the geodesics in Fig. 4.

As for the full dynamics of the $\mathrm{M}_{6}$ cluster, the competition between the internal centrifugal force and the potential force can be captured in Fig. 6, where the energy dependence of the long-time averages of the three gyration radii is shown for each isomer. In the low energy range and in the OCT isomer, the averaged values of the three gyration radii are close to the values at the potential minimum, $a_{1}=a_{2}=a_{3}$ $=5.97$. This means that the cluster is confined close to compact and symmetric mass distributions because of the dominance of the potential force over the internal centrifugal 
force. However, as the total energy increases, the effect of the internal centrifugal force becomes more significant because the quasivelocities tend to be more significant. As a result, the average values of the gyration radii tend to separate from each other [see Fig. 6(a)]. In other words, the OCT isomer is highly elongated in the high energy range. The original isotropic mass distribution of the OCT isomer becomes less preferable as internal energy increases. A similar tendency is observed in the CTBP isomer in Fig. 6(b), where the averaged values of the three gyration radii also tend to separate as the energy increases. However, the elongation effect of the internal centrifugal force in the CTBP isomer is not as significant as in the OCT isomer since the CTBP isomer has an elongated mass distribution already at the potential minimum and the internal centrifugal force is much weaker there as can be seen in Fig. 5(d).

\section{Averaged field of the internal centrifugal force and that of the potential force along a one-dimensional reaction path}

As we have seen in Fig. 3(b), the hopping of the trajectory in the space of gyration radii from one region to the other is essential for the isomerization of the full system. Therefore, the precise characterization of the competition between the internal centrifugal force and the potential force in the space of gyration radii is crucial for understanding the mechanism of the isomerization. We here deduce an averaged field of the internal centrifugal force and that of the potential force numerically along a one-dimensional reaction path introduced in the space of gyration radii.

As a convenient reaction path for the isomerization dynamics, one might consider using the steepest descent path projected onto the space of gyration radii, which is shown in Fig. 4. However, the trajectories in the space of gyration radii do not frequently get close to the projection of the steepest descent path, especially in the high energy range. This can be understood in view of Fig. 6, where the averaged values of gyration radii shift from their values at the potential minimum due to the effect of the internal centrifugal force. In this way, the region of the space of gyration radii densely populated with trajectories, as shown in Fig. 3(b), gradually moves away from the steepest descent path as total energy of the system increases. This indicates that the steepest descent path is not necessarily the best choice of a reaction path.

The reaction path of this study is defined by a direct averaging of the trajectories in the space of gyration radii. As can be seen from Fig. 3(b), the region densely populated by trajectories in the space of gyration radii lies roughly along the $a_{1}$ axis. Therefore, $a_{1}$ can assist in the appropriate averaging. We first set up a lot of equally separated planes in the space of gyration radii that are normal to the $a_{1}$ axis. Large numbers of trajectories in this space are averaged in each plane in terms of the $a_{2}$ and $a_{3}$ components. We then obtain a representative (averaged) point on each plane (for each $a_{1}$ value). These representative points are connected successively to obtain a single path. This procedure is carried out for different total energies. Figure 7 shows thus defined averaged reaction paths in the space of gyration radii for three representative total energies, $E=-11.2 \varepsilon,-10.0 \varepsilon,-8.5 \varepsilon$. In

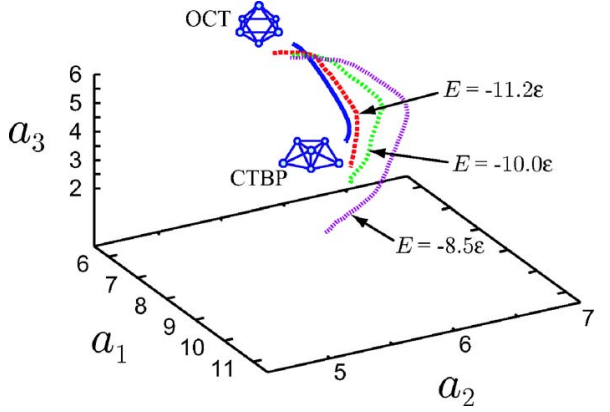

FIG. 7. (Color online) The three broken curves are the averaged reaction paths in the space of gyration radii at total energy $E=-11.2 \varepsilon, E=-10.0 \varepsilon$, and $E=-8.5 \varepsilon$, respectively, as indicated. For comparison, the projection of the steepest descent path onto the same space is shown by the solid curve.

the figure, the projected steepest descent path is also shown for comparison. (The arc-length parameter in Fig. 1 is the length of the steepest descent path in Fig. 7.) It is clear that the arc length of the averaged path becomes longer as energy increases. This is because the trajectories in the space sample a more extensive region as the total energy increases. It is also evident that the averaged reaction path tends to be shifted from the steepest descent path as the total energy increases. Trajectories roughly follow these averaged reaction paths at each total energy when the system undergoes isomerization.

The competition between the internal centrifugal force and the potential force can now be characterized along the averaged reaction paths in Fig. 7. As has been confirmed in Fig. 5, these two forces oscillate rapidly as compared with the time scale of variation of gyration radii. Therefore, it is reasonable to estimate averaged force fields along the reaction paths in the space of gyration radii in the following way. We first introduce a lot of "check points," which are small spheres with a constant radius of 0.03 , along the reaction path in Fig. 7 for each total energy. We then create a representative sample of many trajectories for the same total energy. Every time that the trajectory in the space of gyration radii visits one of the small spheres, the three components of the instantaneous potential force and those of the instantaneous internal centrifugal force are recorded there. These data are finally averaged in the neighborhood of each check point to obtain the averaged potential force,

$\left(\left\langle f_{\mathrm{pot}, 1}\right\rangle,\left\langle f_{\mathrm{pot}, 2}\right\rangle,\left\langle f_{\mathrm{pot}, 3}\right\rangle\right)=\left(\left\langle-\frac{\partial V}{\partial a_{1}}\right\rangle,\left\langle-\frac{\partial V}{\partial a_{2}}\right\rangle,\left\langle-\frac{\partial V}{\partial a_{3}}\right\rangle\right)$,

and the averaged internal centrifugal force, $\left(\left\langle f_{\text {cent }, 1}\right\rangle,\left\langle f_{\text {cent }, 2}\right\rangle,\left\langle f_{\text {cent }, 3}\right\rangle\right)$. Here, each $\left\langle f_{\text {cent }, i}\right\rangle$ is defined as in Eq. (42) with the bracket $\langle\cdot\rangle$ representing the direct numerical average this time. It should be noted that these averages showed a fairly quick convergence in our numerical computations, which supports the validity of our strategy of averaging.

In Fig. 8, shown with arrows are (a) the averaged field of the internal centrifugal force and (b) that of the potential force along the reaction path at total energy $E=-10.0 \varepsilon$ as an illustrative example. In the figures, the reaction path at this 
(a)

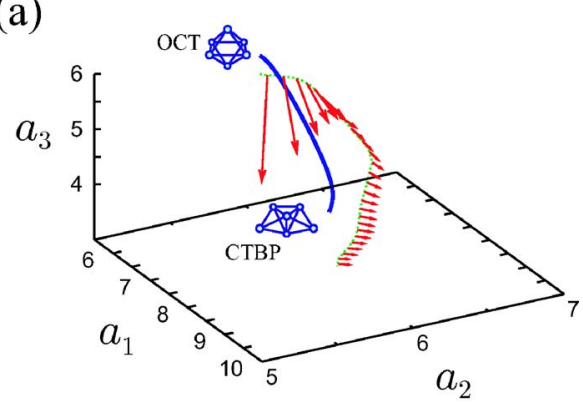

(b)

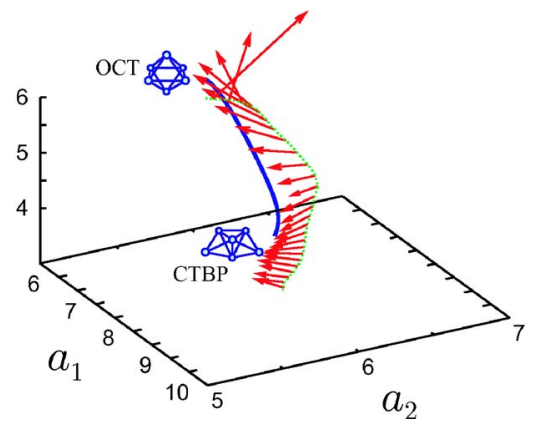

FIG. 8. (Color online) Averaged field of the internal centrifugal force [panel (a)] and that of the potential force [panel (b)] along the reaction path at $E$ $=-10.0 \varepsilon$. The thin broken lines in the two figures constitute the averaged reaction path at $E=-10.0 \varepsilon$ introduced in Fig. 7. The arrows represent the directionality and the magnitude of respective forces at each point along the reaction path. The thick solid curve is the projection of the steepest descent path onto the space for comparison.

total energy is shown by the thin broken curve, which is the same as the one in Fig. 7, and the steepest descent path is shown by the thick solid curve for comparison. The directionality of the averaged field of the internal centrifugal force is essentially the same as that in Fig. 4. Therefore the averaged field of internal centrifugal force is roughly directed from the region of the OCT isomer to that of the CTBP isomer. Furthermore, the internal centrifugal force is stronger in the OCT region than in the CTBP region, which is consistent with the results of Fig. 5(d). This indicates that the internal centrifugal force strongly "pushes" the system from the OCT region to the CTBP region. On the other hand, the averaged potential force field along the reaction path in Fig. 8(b) exhibits a change in direction, roughly pointing, locally, to both points corresponding to the potential minima of the OCT and the CTBP isomer. In other words, the potential force is directed towards the minima of the potential wells in the reduced space of gyration radii as is naturally expected. To summarize, the averaged potential force works to keep the internal mass distribution of the cluster close to that of each potential minimum structure, while the averaged internal centrifugal force has a persistent tendency to bring the system mass distribution from that of the OCT isomer to that of the CTBP isomer.

It should also be noted that the reaction path has been shifted from the steepest descent path roughly in the direction of the internal centrifugal force. Therefore the results of Figs. 8(a) and 8(b) indicate that the shift of the reaction path is determined by the balance between the internal centrifugal force and the potential force. We computed the averaged force fields for different total energies in a way similar to
Fig. 8: The directionality of the two force fields does not change significantly depending on the total energy. However, the relative strength between the internal centrifugal force and the potential force along the reaction path changes markedly depending on the total energy. This will be quantified in the next subsection in terms of the line integrals (effective energies) of these forces.

\section{Switching mechanism between isomers}

The existence of the field of the internal centrifugal force in Fig. 8(a) indicates that an extra amount of work is needed in order for the vibrating cluster to change its gyration radii along the reaction path from the CTBP conformation to the OCT conformation. This work is different from the work necessary to overcome the potential forces in Fig. 8(b). Here, these two works are quantified by considering the line integrals of the two forces along the reaction path. We then present a possible rationalization for the energy-dependent switching of the favored mass distribution of the $\mathrm{M}_{6}$ cluster in terms of the competition between the internal centrifugal force and the potential force. This will explain the switching of the structural preference presented in Sec. II B.

We first characterize the energy-dependent switching of the structural preference of the cluster along the reaction paths introduced in Fig. 7. Similar to the method in Sec. IV C, a number of small spheres with constant radius 0.03 are first introduced along the reaction path. At each total energy, we count the total number of times that the trajectories visit each small sphere during long transients for a large enough ensemble of trajectories. These numbers are normalized to unity to obtain a probability distribution along each reaction path. The upper three panels of Fig. 9 show thus obtained probability distributions along the reaction paths for different energies, (a) $E=-11.2 \varepsilon$, (b) $E=-10.0 \varepsilon$, and (c) $E$ $=-8.5 \varepsilon$. The horizontal axis of each of these figures is the arc length along each reaction path in the space of gyration radii, which serves as the reaction coordinate. The left part (the short arc-length part) of each figure corresponds to the region of the OCT isomer, while the right part corresponds to the region of the CTBP isomer. Since the reaction path becomes longer as the total energy increases (see Fig. 7), the width of these panels increases from (a) to (c). In each panel, two peaks are observed in the probability distribution, the left one of which corresponds to the OCT isomer while the right one corresponds to the CTBP isomer. At low total energy [Fig. 9(a)], the left peak is higher than the right peak. This indicates that the system prefers to be in the mass distribution of the OCT isomer than that of CTBP. On the other hand, as the total energy increases [Figs. 9(b) and 9(c)], the left peak is lowered and the right peak becomes higher. In other words, the preferable mass distribution of the cluster switches from that of the OCT isomer to that of the CTBP isomer. In this way, the switching of the structural preference observed in Sec. II B is explained in terms of the reaction paths in Fig. 7.

The above argument of the switching in the probability distribution is analogous to first-order-like phase transitions observed in clusters ${ }^{51-53}$ and polymers. ${ }^{54,55}$ In these systems, 
(a)
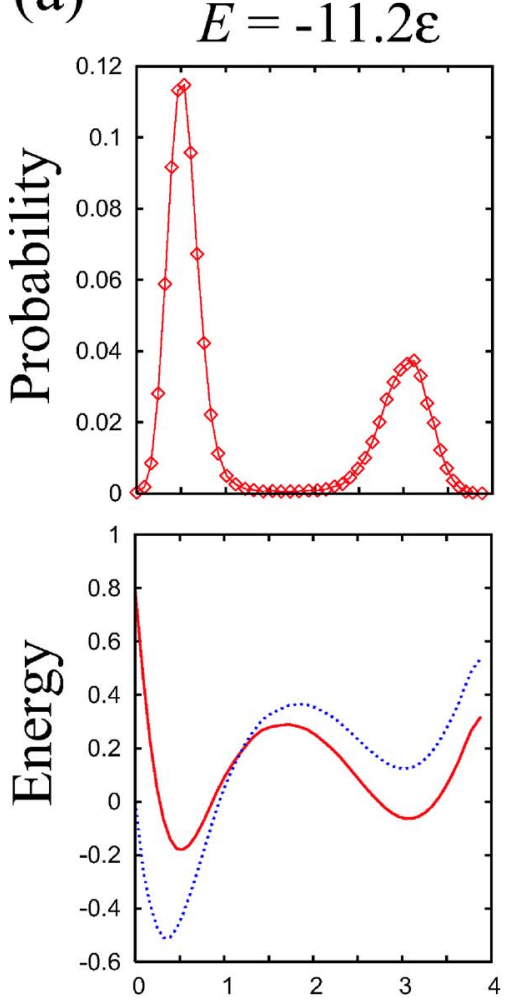

Reaction coordinate (b)
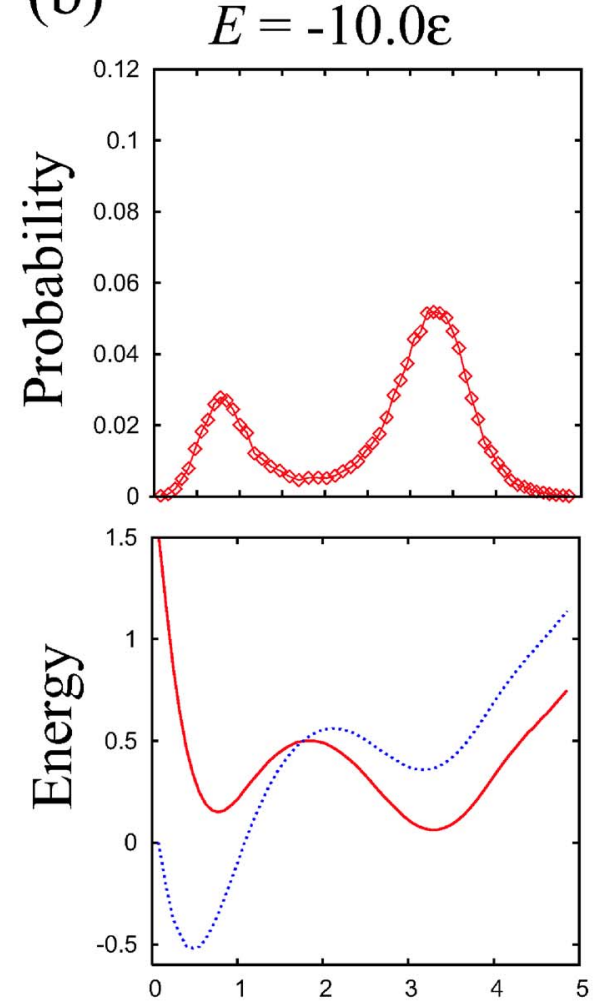

Reaction coordinate (c)
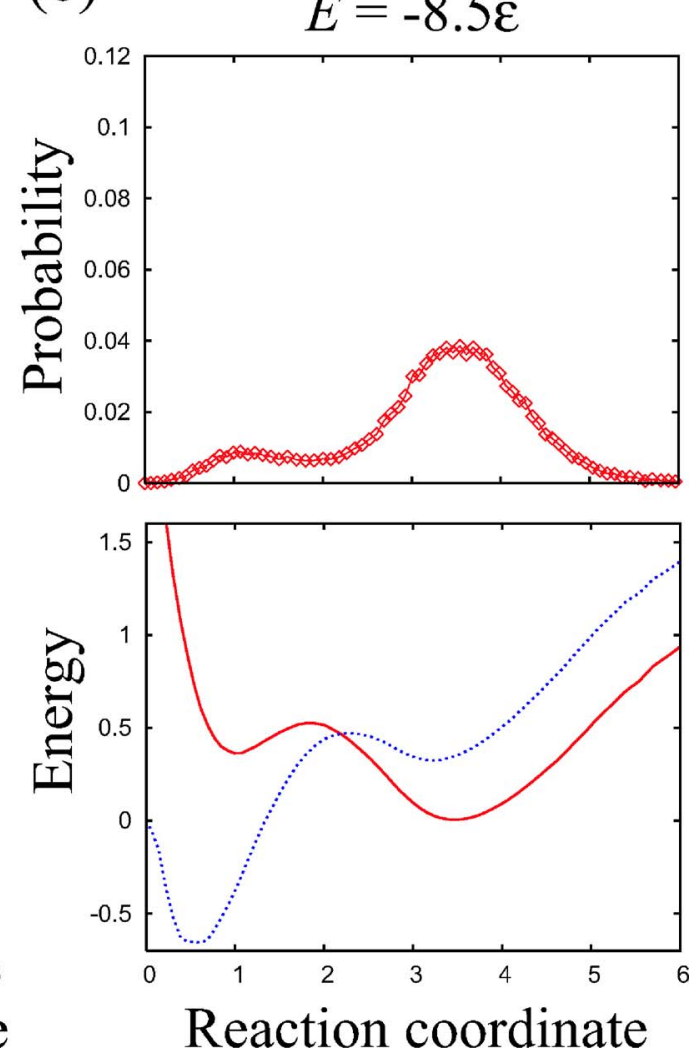

FIG. 9. (Color online) The upper three panels represent the probability distribution along the reaction coordinates introduced in Fig. 7 at total energy (a) $E=-11.2 \varepsilon$, (b) $E=-10.0 \varepsilon$, and (c) $E=-8.5 \varepsilon$. The lower three panels represent the corresponding reconstructed potential energy $V_{\text {pot }}($ dotted line) and the superposed energy $V_{\text {pot+cent }}$ (solid line) along the reaction coordinates.

equilibrium probability density plotted along the axis of an order parameter shows a bimodal distribution in the critical energy or temperature range. In our argument, the reaction coordinate introduced in the space of gyration radii plays the role of an order parameter. And the probability distribution along the reaction coordinate does exhibit this switching. Following the classification by Davis et al. for an $\mathrm{Ar}_{13}$ cluster, ${ }^{52}$ OCT can be regarded as the solidlike form of the cluster while CTBP as its liquidlike form.

Now, the mechanism of switching to the favored isomer can be rationalized in terms of the work necessary to move the system against the averaged force fields obtained in Figs. 8 (a) and 8(b). The line integral of the negative of the averaged potential force field along the reaction path gives a reconstructed potential energy curve $V_{\text {pot }}$ as

$$
V_{\mathrm{pot}}=-\int_{\text {path }} \sum_{i=1}^{3}\left\langle f_{\mathrm{pot}, i}\right\rangle d a_{i} .
$$

We carried out this line integral numerically starting at one of the end points of the reaction path, where the $a_{1}$ is the smallest. Since the absolute value of the potential energy is arbitrary, we set $V_{\text {pot }}$ to zero at the starting point of the line integral. The potential $V_{\text {pot }}$ is essentially the reduced potential energy curve that the gyration radii feel along the reaction coordinate. In the lower three panels of Fig. 9, $V_{\text {pot }}$ is shown for the three representative total energy values, $E=$ $-11.2 \varepsilon,-10.0 \varepsilon$, and $-8.5 \varepsilon$, with dotted curves. The essential topography of $V_{\text {pot }}$ does not change significantly depending on the total energy. That is, $V_{\text {pot }}$ always has two wells. The left well corresponds to the OCT isomer while the right one corresponds to the CTBP isomer. The well for the OCT isomer is always much deeper than that for the CTBP isomer. The potential $V_{\text {pot }}$ resembles the original potential energy curve along the steepest descent path shown in Fig. 1. The curve of $V_{\text {pot }}$ is obviously not sufficient for explaining the energy-dependent change of the probability distribution along the reaction paths shown in the upper panels of Fig. 9.

We next superpose the effect of the internal centrifugal force over that of the potential curve $V_{\text {pot }}$. Similar to Eq. (51), a superposed line integral of the averaged potential force and the averaged internal centrifugal force is introduced as

$$
V_{\text {pot+cent }}=-\int_{\text {path }} \sum_{i=1}^{3}\left(\left\langle f_{\text {pot }, i}\right\rangle+\left\langle f_{\text {cent }, i}\right\rangle\right) d a_{i} .
$$

The solid curves in the lower three panels of Fig. 9 are the superposed energy $V_{\text {pot+cent }}$ Similar to the curve of $V_{\text {pot }}$, this energy curve $V_{\text {pot+cent }}$ shows two wells corresponding to the two isomers. However, the topography of $V_{\text {pot+cent }}$ changes dramatically depending on the total energy of the system. At low total energy, $E=-11.2 \varepsilon$, the left well of $V_{\text {pot+cent }}$, which corresponds to the OCT isomer, is deeper than the right well that corresponds to the CTBP isomer. As the total energy increases, the left well gets shallower while the right well 
gets deeper. This is because the effect of the internal centrifugal force becomes more significant as the total energy of the dynamics increases, while the potential curve $V_{\text {pot }}$ does not change significantly. Note that the internal centrifugal force has the directionality to "push" the system from the OCT region to the CTBP region as confirmed in Fig. 4(a) and in Fig. 8(a). In this way, the OCT isomer becomes less stable dynamically while the CTBP isomer becomes more preferred as the total energy increases. This dramatic change of the superposed energy curve $V_{\text {pot+cent }}$ explains the change of the probability distribution in the upper panels of Fig. 9 fairly well. To summarize, the predominance of the OCT isomer at low energy is mainly supported by the predominance of the potential force, while the predominance of the CTBP isomer at high energy is mainly supported by the predominance of the internal centrifugal force.

\section{CONCLUDING REMARKS}

We have presented a methodology that enhances our understanding of the mechanism of large-amplitude structural transitions of isolated molecules in terms of the reduced dynamics of three molecular gyration radii. The framework of geometric mechanics together with the use of principal-axis hyperspherical coordinates has provided the basis for a systematic dimensionality reduction. After a careful separation of rotational degrees of freedom, the $(3 n-6)$ internal degrees of freedom of an $n$-atom system were decomposed into the three gyration radii and the $(3 n-9)$ hyperangular modes. It has been shown empirically that the three gyration radii of a cluster serve as slow collective variables while the remaining hyperangular modes serve as internal "bath" modes. Based on this approximation, the dependence of the equations of motion for the gyration radii on the hyperangular modes was averaged out. When this approximation is valid, the present method can be a useful tool to explore the essential mechanism of large-amplitude motions of molecules in terms of the reduced dynamics of only the three gyration radii irrespective of the total number of degrees of freedom of the system.

The reduced equations of motion for the gyration radii have revealed a competition between a kinematic force and the potential force in the large-amplitude motions of the cluster. The kinematic force is identified as an internal centrifugal force arising from the dynamical coupling of gyration radii with the hyperangular modes via the intrinsic nonEuclidean metric of the internal space. This internal centrifugal force has the remarkable effect of elongating the symmetric mass distribution of the system as well as an effect of inflating the system. These effects often counteract the effect of the potential force, which generally serves to keep the mass distribution of the system symmetric and compact. The competition between these two forces has turned out to be important in determining the most preferable conformation of the cluster at different total energies: In the low energy range, the $\mathrm{M}_{6}$ cluster prefers to be in the OCT conformation, which has a compact and symmetric mass distribution, because of the dominance of the effect of the potential force. On the other hand, in the high energy range, the CTBP conformation, which has a distorted (elongated) mass distribu- tion, becomes more preferable due to the dominance of the effect of the internal centrifugal force. This switching of the structural preference has been compactly characterized by an effective energy curve along a one-dimensional reaction path introduced in the space of gyration radii.

The elongation and the inflation effects of the internal centrifugal force should be of general importance in a wide class of molecular reactions (in vacuum), where the system significantly changes its mass distribution. For example, because of the directionality of this force, its roles should be important in dissociation and recombination reactions. While the potential function is specific to the system of interest, the kinetic energy expressions such as Eqs. (35) and (38) are common to general $n$-atom molecules. Therefore the internal centrifugal force terms in Eqs. (39)-(41) also arise ubiquitously in the dynamics of general $n$-atom molecules. The present study as well as another recent one ${ }^{60}$ suggest that certain extra "work" may be needed in addition to the work necessary for overcoming the potential barrier when a vibrating molecule changes its mass distribution. This extra work can be determined by the averaged fields of the internal centrifugal force deduced in the space of gyration radii such as in Fig. 4(a) and in Fig. 8(a). It can be generally said that the structural transitions against the field of the internal centrifugal force require this extra work. These reactions are, therefore, harder than would be expected from the potential barrier only. On the other hand, the reactions that respect the directionality of the internal centrifugal force will be correspondingly easier than expected. For example, our illustrative $\mathrm{M}_{6}$ cluster experiences the extra "barrier" in reacting from the CTBP conformation to the OCT conformation since the internal centrifugal force works against this transition. Since the rearrangement of mass distribution is a common feature of a variety of molecular reaction dynamics, this kind of mass-related kinematic barriers should shed new light on the conventional picture of reaction mechanisms.

In this paper, we have focused on the dynamics of the three gyration radii as collective variables based on the approximation of the time scale separation between the gyration radii and the hyperangular modes. It is thereby another important issue to clarify the precise physical origin of the time scale separation itself. Since the gyration radii are associated with the overall (collective) deformation of a molecule, they should be closely related to the low-frequency normal modes. The hyperangular modes, on the other hand, are rather sensitive to the local changes of molecular conformation and would have large overlaps with the highfrequency normal modes. In this way, comparison with the normal-mode picture will provide us with deeper insights into the origin of the time scale separation. The equations of motion for the hyperangular modes would also be useful to highlight the qualitative difference of their dynamics from that of the gyration radii. Indeed, the structure of the equations of motion for the hyperangular modes ${ }^{61}$ is largely different from those for the gyration radii. The most striking difference is that the kinematic terms in the equations of motion for the hyperangular modes appear to vanish after averaging. This indicates that significant kinematic effects arise not in the dynamics of hyperangular modes but in the 
dynamics of the three gyration radii. In this case, our approach in this paper of focusing on the dynamics of the three gyration radii will be further strengthened.

The present study has assumed an isolated system with zero total angular momentum, which is the most elementary molecular system. It is therefore important, for generality, to extend the present framework to systems in different environments step by step. The next elementary system is the molecules with nonzero total angular momentum, in which the kinematic forces such as the (normal) centrifugal force and the Coriolis force also influence the mass distribution of the system in addition to the internal centrifugal force of this study. Effects of the former two forces may also be characterized in the three-dimensional space of gyration radii, as in Figs. 8(a) and 8(b). It is also an interesting issue to extend the present approach to systems in thermal environments such as in a heat bath or in solvents. Since the internal centrifugal force has a general tendency to become strong in high energy (or temperature) range and has an effect of inflating and elongating the system, this force is naturally expected to act as a driving force for a wide class of largeamplitude motions and phase transitions including protein (un)folding. Finally, another interesting issue is to numerically evolve the low-dimensional system of the gyration radii along the line of the coarse-molecular dynamics. ${ }^{6,7}$ This will make it possible to explore the free energy topography as well as the reaction rates of complex molecular systems. These issues will be scrutinized in our future work.

\section{ACKNOWLEDGMENTS}

The authors thank Aron Kuppermann and Kazuo Takatsuka for valuable discussions. This work was partially supported by NSF-ITR Grant No. ACI-0204932, ICB-ARO Grant No. DAAD19-03-D-0004, and NSF-DMS-0505711. One of the authors (T.Y.) has also been supported by JSPS Research Fellowships for Young Scientists. Another author (I.G.K.) gratefully acknowledges the support of a Guggenheim Fellowship.

\section{APPENDIX: EIGENVALUES AND EIGENVECTORS}

We here summarize the relationship among the vectors and the singular values $\left\{\boldsymbol{e}_{i}\right\},\left\{\boldsymbol{u}_{i}\right\},\left\{a_{i}\right\}(i=1,2,3)$ in Eqs. (7)-(9). The relationship between the gyration radii and the principal moments of inertia, Eq. (11), is also shown.

The vectors and the singular values $\left\{\boldsymbol{e}_{i}\right\},\left\{\boldsymbol{u}_{i}\right\},\left\{a_{i}\right\}(i$ $=1,2,3)$ in Eqs. (7)-(9) are mutually related by the following eigenvalue equations: ${ }^{31-33,35}$

$$
\begin{aligned}
& \left(\mathbf{W}_{s} \mathbf{W}_{s}^{T}\right) \boldsymbol{e}_{i}=a_{i}^{2} \boldsymbol{e}_{i} \quad(i=1,2,3), \\
& \left(\mathbf{W}_{s}^{T} \mathbf{W}_{s}\right) \boldsymbol{u}_{i}=a_{i}^{2} \boldsymbol{u}_{i} \quad(i=1,2,3) .
\end{aligned}
$$

That is, the vectors $\boldsymbol{e}_{1}, \boldsymbol{e}_{2}$, and $\boldsymbol{e}_{3}$ are the eigenvectors of the $3 \times 3$ symmetric matrix $\mathrm{W}_{s} \mathrm{~W}_{s}^{T}$, whereas the vectors $\boldsymbol{u}_{1}, \boldsymbol{u}_{2}$, and $\boldsymbol{u}_{3}$ are the eigenvectors of the $(n-1) \times(n-1)$ symmetric matrix $\mathbf{W}_{s}^{T} \mathbf{W}_{s}$. The matrices $\mathbf{W}_{s} \mathbf{W}_{s}^{T}$ and $\mathbf{W}_{s}^{T} \mathbf{W}_{s}$ have the common eigenvalues $a_{1}^{2}, a_{2}^{2}$, and $a_{3}^{2}$, the squares of gyration radii. The matrix $\mathrm{W}^{T} \mathrm{~W}$ has $(n-4)$ zero eigenvalues that correspond to the eigenvectors $\boldsymbol{u}_{4}, \ldots, \boldsymbol{u}_{n-1}$ in Eq. (9).
The gyration radii, $a_{1}, a_{2}, a_{3}$, and the principal moments of inertia, $M_{1}, M_{2}, M_{3}$, are closely related as follows. The moment of inertia tensor $\mathrm{M}_{s}$ (with respect to the space-fixed frame) is first expressed using the matrix $W_{s}$ in Eq. (5) as

$$
\mathrm{M}_{s}=\operatorname{Tr}\left(\mathrm{W}_{s} \mathrm{~W}_{s}^{T}\right) \mathrm{I}-\mathrm{W}_{s} \mathrm{~W}_{s}^{T},
$$

where $\operatorname{Tr}(\cdot)$ represents the trace of the matrix and $\mathrm{I}$ is a 3 $\times 3$ unit matrix. By inserting $\mathrm{W}_{s}=\mathrm{RNU}^{T}$ of Eq. (6) into Eq. (A3) and using Eq. (8), one obtains

$$
\mathrm{R}^{T} \mathrm{M}_{s} \mathrm{R}=\left(\begin{array}{ccc}
a_{2}^{2}+a_{3}^{2} & 0 & 0 \\
0 & a_{1}^{2}+a_{3}^{2} & 0 \\
0 & 0 & a_{1}^{2}+a_{2}^{2}
\end{array}\right) \equiv \mathrm{M}
$$

which means the matrix $\mathrm{R}$ diagonalizes the moment of inertia tensor $\mathrm{M}_{s}$. This justifies that $\mathrm{R}$ in Eq. (6) is the principalaxis frame. Equation (A4) also proves the relationship between the gyration radii and the principal moments of inertia, Eq. (11). In Eq. (A4) we have introduced the diagonalized moment of inertia tensor $\mathrm{M}$, which is the moment of inertia tensor with respect to the principal-axis frame.

${ }^{1}$ R. Zwanzig, Phys. Rev. 124, 983 (1961).

${ }^{2}$ H. Mori, Prog. Theor. Phys. 33, 423 (1965).

${ }^{3}$ R. Zwanzig, Nonequilibrium Statistical Mechanics (Oxford University Press, New York, 2001).

${ }^{4}$ A. Laio and M. Parrinello, Proc. Natl. Acad. Sci. U.S.A. 99, 12562 (2002).

${ }^{5}$ R. Elber, A. Cárdenas, A. Ghosh, and H. A. Stern, Adv. Chem. Phys. 126, 93 (2003).

${ }^{6}$ G. Hummer and I. G. Kevrekidis, J. Chem. Phys. 118, 10762 (2003).

${ }^{7}$ D. I. Kopelevich, A. Z. Panagiotopoulos, and I. G. Kevreidis, J. Chem. Phys. 122, 044907 (2005); 122, 044908 (2005).

${ }^{8}$ W. H. Miller, N. C. Handy, and J. E. Adams, J. Chem. Phys. 72, 99 (1980).

${ }^{9}$ P. G. Bolhuis, D. Chandler, C. Dellago, and P. L. Geissler, Annu. Rev. Phys. Chem. 53, 291 (2002).

${ }^{10}$ W. E, W. Ren, and E. Vanden-Eijnden, Chem. Phys. Lett. 413, 242 (2005).

${ }^{11}$ P. J. Flory, Principles of Polymer Chemistry (Cornell University Press, Ithaca, NY, 1953).

${ }^{12}$ M. Doi and S. F. Edwards, The Theory of Polymer Dynamics (Oxford University Press, New York, 1986).

${ }^{13}$ M. P. Taylor, J. Chem. Phys. 114, 6472 (2001).

${ }^{14}$ J. M. Polson and N. E. Moore, J. Chem. Phys. 122, 024905 (2005).

${ }^{15}$ F. B. Sheinerman and C. L. Brooks III, J. Mol. Biol. 278, 439 (1998).

${ }^{16}$ T. Uzawa, S. Akiyama, T. Kimura, S. Takahashi, K. Ishimori, I. Morishima, and T. Fujisawa, Proc. Natl. Acad. Sci. U.S.A. 101, 1171 (2004).

${ }^{17}$ H. Noguchi and K. Yoshikawa, J. Chem. Phys. 109, 5070 (1998); 113, 854 (2000).

${ }^{18}$ F. Calvo and P. Labastie, Eur. Phys. J. D 3, 229 (1998).

${ }^{19}$ P. Parneix and F. Calvo, J. Chem. Phys. 121, 11088 (2004).

${ }^{20}$ J. E. Marsden, T. S. Ratiu, and J. Scheurle, J. Math. Phys. 41, 3379 (2000).

${ }^{21}$ J. E. Marsden and T. S. Ratiu, Introduction to Mechanics and Symmetry (Springer, New York, 1999).

${ }^{22}$ M. Kummer, Indiana Univ. Math. J. 30, 281 (1981).

${ }^{23}$ J. E. Marsden, R. Montgomery, and T. Ratiu, Contemp. Math. 28, 101 (1984).

${ }^{24}$ A. Guichardet, Ann. Inst. Henri Poincare, Sect. A 40, 329 (1984).

${ }^{25}$ T. Iwai, Ann. Inst. Henri Poincare, Sect. A 47, 199 (1987).

${ }^{26}$ A. Tachibana and T. Iwai, Phys. Rev. A 33, 2262 (1986).

${ }^{27}$ R. G. Littlejohn and M. Reinsch, Rev. Mod. Phys. 69, 213 (1997).

${ }^{28}$ C. Eckart, Phys. Rev. 46, 383 (1934).

${ }^{29}$ X. Chapuisat and A. Nauts, Phys. Rev. A 44, 1328 (1991).

${ }^{30}$ X. Chapuisat, Phys. Rev. A 45, 4277 (1992).

${ }^{31}$ A. Kuppermann, Adv. Mol. Vib. Collision Dyn. 2B, 117 (1993).

${ }^{32}$ A. Kuppermann, J. Phys. Chem. 100, 2621 (1996).

${ }^{33}$ A. Kuppermann, J. Phys. Chem. 101, 6368 (1997). 
${ }^{34}$ V. Aquilanti and S. Cavalli, J. Chem. Phys. 85, 1355 (1986).

${ }^{35}$ R. G. Littlejohn and M. Reinsch, Phys. Rev. A 52, 2035 (1995).

${ }^{36}$ R. G. Littlejohn, K. A. Mitchell, V. A. Aquilanti, and S. Cavalli, Phys. Rev. A 58, 3705 (1998).

${ }^{37}$ R. G. Littlejohn, K. A. Mitchell, M. Reinsch, V. Aquilanti, and S. Cavalli, Phys. Rev. A 58, 3718 (1998).

${ }^{38}$ V. Aquilanti, A. Lombardi, and E. Yurtsever, Phys. Chem. Chem. Phys. 4, 5040 (2002).

${ }^{39}$ V. Aquilanti, A. Lombardi, M. B. Sevryuk, and E. Yurtsever, Phys. Rev. Lett. 93, 113402 (2004).

${ }^{40}$ V. Aquilanti, A. Lombardi, and M. B. Sevryuk, J. Chem. Phys. 121, 5579 (2004).

${ }^{41}$ T. Yanao and K. Takatsuka, Phys. Rev. A 68, 032714 (2003).

${ }^{42}$ T. Yanao and K. Takatsuka, J. Chem. Phys. 120, 8924 (2004).

${ }^{43}$ T. Yanao and K. Takatsuka, Adv. Chem. Phys. 130, 87 (2005).

${ }^{44}$ V. Aquilanti, S. Cavalli, and M. B. Sevryuk, J. Math. Phys. 35, 536 (1994).

${ }^{45}$ D. Blume and C. H. Greene, J. Chem. Phys. 112, 8053 (2000).

${ }^{46}$ O. Sørensen, D. V. Fedorov, and A. S. Jensen, Phys. Rev. A 66, 032507 (2002).
${ }^{47}$ O. Sørensen, D. V. Fedorov, and A. S. Jensen, Phys. Rev. A 70, 013610 (2004).

${ }^{48}$ R. S. Berry, Chem. Rev. (Washington, D.C.) 93, 2379 (1993).

${ }^{49}$ P. A. Braier, R. S. Berry, and D. J. Wales, J. Chem. Phys. 93, 8745 (1990).

${ }^{50}$ T. Komatsuzaki and R. S. Berry, J. Chem. Phys. 110, 9160 (1999); 115, 4105 (2001)

${ }^{51}$ J. Jellinek, T. L. Beck, and R. S. Berry, J. Chem. Phys. 84, 2783 (1986).

${ }^{52}$ H. L. Davis, J. Jellnek, and R. S. Berry, J. Chem. Phys. 86, 6456 (1987).

${ }^{53}$ P. Labastie and R. L. Whetten, Phys. Rev. Lett. 65, 1567 (1990).

${ }^{54}$ Y. Zhou, C. K. Hall, and M. Karplus, Phys. Rev. Lett. 77, 2822 (1996).

${ }^{55}$ M. Takano, T. Takahashi, and K. Nagashima, Phys. Rev. Lett. 80, 5691 (1998).

${ }^{56}$ F. G. Amar and R. S. Berry, J. Chem. Phys. 85, 5943 (1986).

${ }^{57}$ C. Seko and K. Takatsuka, J. Chem. Phys. 104, 8613 (1996).

${ }^{58}$ M. A. Miller and D. J. Wales, J. Chem. Phys. 107, 8568 (1997).

${ }^{59}$ G. Strang, Linear Algebra and Its Applications (Academic, New York, 1976).

${ }^{60}$ T. Yanao, W. S. Koon, and J. E. Marsden, Phys. Rev. A 73, 052704 (2006).

${ }^{61}$ T. Yanao, W. S. Koon, J. E. Marsden, and I. G. Kevrekidis (unpublished). 